\title{
Der verflixte italienische Dativ - ein (erster) semantisch-grammatikalischer Zähmungsversuch
}

\author{
ALEXANDRA KRATSCHMER \\ Institut für Sprache, Literatur und Kultur, Aarhus Universität, Dänemark
}

\begin{abstract}
Angående nogle bestemte italienske epistemiske og deontisk/aletiske prædikater kan der konstateres en semantisk forskel mellem prædikater med tilføjelser i form af dativiske klitiske pronominer eller præpositionssyntagmer på basis af præpositionen $a$ på den ene side og tilføjelser $i$ form af præpositionssyntagmer på basis af præpositionen per på den anden side. På det epistemiske område kan der skelnes mellem fx mi è chiaro og a me è chiaro (ordret "mig er det klart"), som har en læsning (io) so "jeg ved", og per me è chiaro "for mig er det klart", der har en læsning (io) sono convinto "jeg er overbevist". På det deontisk/aletiske område ligger forskellen derimod i præsentationen af det dativiske modale subjekt som enten absolut eller paradigmatiseret via emfase. Der kan skelnes mellem fx mi è necessario og a me è necessaio (ordret "mig er det nødvendigt"), som har en læsning (io) [udeladt eller svagt betonet] sono costretto "jeg er nødsaget", og per me è necessario "for mig er det nødvendigt", der har en læsning io [med emfatisk intonation] sono costretto "der er nogen (deriblandt mig), som er nødsaget".
\end{abstract}

Med henblik på en tilstræbt tilnærmelse til en koncis definition af begrebet "semantisk dativ" og den dertil nødvendige systematiske bekrivelse og klassifikation af de "dativiske" semantiske roller, som nogle sætningsled kan indehave, kan det noteres, at for de indtil videre undersøgte italienske "endosubjektive" prædikater (prædikater, der udtrykker et neurokognitivt forhold i et bevidst subjekt) gælder, at de - ikke overraskende tillader en polyfon analyse. Inden for en sådan polyfon analyse kan de endosubjektive prædikaters indhold formaliseres som polyfon konfiguration, hvor flere diskursindivider præsenteres som kilder til hver deres synspunkt. I modsætning til den oprindelige forskningshypotese kan forskellen vedr. de dativiske tilføjelsers semantiske rolle ved de endosubjektive prædikater dog ikke tilskrives et enkelt diskursindivid 
inden for den polyfone konfiguration, men kræver snarere en beskrivelse, hvor de forskellige semantiske roller giver tolkningsinstrukser vedrørende - forskellige polyfone helkonfigurationer.

\section{Die BeGRIFFE "DATIV" UND "DATIVISCH"}

Im Italienischen existiert eine lange Reihe an Prädikaten, welche sich mit "dativischen" Größen verbinden lassen. Der Begriff "dativisch" kann einerseits als morphologischer Begriff aufgefasst werden und bezeichnet in diesem Fall eine Nominalbeugung. Dies kennt man von Sprachen mit morphologischem Kasus wie etwa Latein und Deutsch, welche morphologischen Dativ sowohl im substantivischen als auch im pronominalen Kasusparadigma aufweisen, oder von den romanischen Sprachen, welche diese Beugung ausschließlich im pronominalen Bereich bewahrt haben (z.B. italienisch gli/le "ihm/ihr"). Der Begriff "dativisch" kann jedoch auch als semantischer Begriff aufgefasst werden, welcher im Augenblick zwar als prätheoretisch angesehen werden muss, welcher jedoch unserer Meinung nach zu einer nützlichen Kategorie werden kann, um eine Reihe an semantischen Phänomenen systematisieren zu helfen.

Wir wollen im vorliegenden Artikel einen ersten Schritt in diese Richtung unternehmen, indem wir einige "dativogene" Prädikate (Prädikate, welche dativische Ergänzungen zulassen) untersuchen wollen, um deren semantische Struktur (welche wir im Übrigen als polyphon ansehen) und die genauere Funktion der dativischen Ergänzungen in diesen Kontexten zu ermitteln.

Wir sehen den Begriff "dativisch" als prätheoretischen semantischen Begriff als einen Überbegriff für eine Gruppe semantischer Rollen an, welche unter anderem (aber nicht ausschließlich) Nominalphrasen zugeschrieben werden können, die mit morphologischer Dativmarkierung versehen sind. In einer Sprache wie dem Italienischen, welche morphologischen Dativ wie gesagt nur bei Pronomen kennt, muss "semantischer Dativ" ebenso die entsprechenden periphrastischen Präpositionalphrasen im nicht-pronominalen Nominalbereich umfassen ( $g l i$ "ihm" > > all'insegnante "dem Lehrer"). Darüberhinaus kann der Begriff in Bezug auf periphrastische Präpositionalphrasen angewandt werden, welche intuitiv eine verwandte semantische Rolle haben (per me, per l'insegnante "für mich", "für den Lehrer"). 


\section{DER VERFLIXTE ITALIENISCHE DATIV - EIN (ERSTER) SEMANTISCH-GRAMMATIKALISCHER \\ ZÄHMUNGSVERSUCH}

Im Hinblick auf eine konzise Definition des Begriffs "semantischer Dativ" soll diese Intuition durch eine systematische Beschreibung und Klassifizierung der relevanten semantischen Rollen objektiviert werden. Aufgrund des nicht geringen Umfangs und der großen internen Variation der italienischen dativogenen Prädikatgruppe wird sich dies wohl als ein längeres Vorhaben erweisen, im Rahmen dessen der vorliegende Beitrag nur als ein erster Schritt gelten kann.

\section{DiE DIDAKTISCHE NOTWENDIGKEIT}

Als wir uns mit den dativischen Ergänzungen zu beschäftigen begannen, geschah dies aus didaktischen Gründen. Wir kamen im Unterrichtsfach "Italienische Sprachbeherrschung" immer wieder in Erklärungsnot, wenn es galt, den Studierenden die richtigen Ergänzungen zu den einzelnen dativogenen Prädikaten beizubringen. Ausgehend von den Generalisierungsfehlern und Hyperkorrektionen der Studierenden war leicht zu erkennen, dass dies ein Gebiet mit akutem Forschungsbedarf war. Kaum hatten sie etwa gelernt, dass man sowohl mi ̀̀ (im)possibile als auch per me ̀̀ (im)possibile "mir/für mich ist es (un)möglich" sagen kann, mussten sie erneut korrigiert und darüber informiert werden, dass es nur mi sembra und nicht *per me sembra "mir scheint" heißen kann oder dass man nicht *è (im)possibile a Catarina, sondern nur è (im)possibile per Catarina "es ist (un)möglich für Catarina" sagen kann.

In den einzelnen Ausdrücken liegen vermutlicherweise verschiedene semantische Nuancen. Gleichzeitig sind die erwähnten und andere grammatikalische Imkompatibilitäten vermutlicherweise auf semantische Inkompatibilitäten zurückzuführen. Die Ermittlung dieser semantischen Gegebenheiten in den fraglichen Ausdrücken muss daher der Schlüssel zur Problematik der dativischen Ergänzungen sein und ist gleichzeitig, wie aus Obigem hervorgeht, auch aus didaktischer Sicht eine Notwendigkeit.

Eine derartige semantische Aufschlüsselung verspricht andererseits aber auch neue theoretische Einsichten in die Natur des semantischen Dativs, wenn die einzelnen Nuancen hoffentlich einmal zu einem übergeordneten Mosaik zusammengesetzt werden können. 


\section{Die itAliensChen DATIVOgenen PrädIKATE}

Innerhalb der italienischen dativogenen Prädikate kann man unterscheiden zwischen Prädikaten, welche mit Subjektsätzen kompatibel sind (1), und Prädikaten, welche dies nicht sind (2):

(1) Mi/per me è chiaro che non piove.

'Mir/Für mich ist klar, dass es nicht regnet.'

(2) Gianni compra un regalo a/per Maria.

'Gianni kauft Maria ein Geschenk/kauft ein Geschenk für Maria.'

In jener Gruppe, welche kompatibel mit Subjektsätzen ist, gibt es die "endosubjektiven" Prädikate (Prädikate, welche einen neurokognitiven Umstand in einem bewussten Subjekt ausdrücken), wie in obigem Satz (1), und nicht"endosubjektive" Prädikate, wie etwa (3):

(3) Mi succede spesso che il computer si blocchi.

'Mir passiert (es) oft, dass der Computer abstürzt.'

Unter den endosubjektiven Prädikaten gibt es Prädikate, welche ein epistemisches Urteil ${ }^{1}$ ausdrücken (siehe (1) oben, hier wiederholt als (4)), Prädikate, welche deontisch/alethische Modalität ausdrücken (Notwendigkeit, Gebot, Verbot, Zulassung; (5)), die Scheinen-Prädikate sembrare/parere (welche epistemische Modalität und Evidentialität ${ }^{2}$ ausdrücken; (6)), Prädikate, welche psychisches Erleben ausdrücken (7) und rein volitive Prädikate ("wissen wollen" oder "haben wollen"; (8)):

(4) Mi/per me è chiaro che non piove.

'Mir/Für mich ist klar, dass es nicht regnet.'

(5) Mi/per me è necessario che tu mi dica la verità.

'Mir/für mich ist (es) notwendig, dass du mir die Wahrheit sagst.'

(6) Mi sembra/pare che tutto sia in ordine.

'Mir scheint, dass alles in Ordnung ist.' 


\section{DER VERFLIXTE ITALIENISCHE DATIV - EIN (ERSTER) SEMANTISCH-GRAMMATIKALISCHER \\ ZÄHMUNGSVERSUCH}

(7) Mi piace che tu sia tornato.

'Mir gefällt/es freut mich, dass du zurückgekommen bist.'

(8) Mi interessa che tu non parta/se (tu) parti.

'Es interessiert mich ( $\cong$ ich will haben), dass du nicht abreist/Es

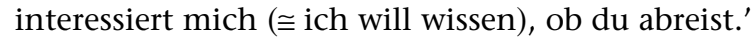

Wir möchten im Anschluss einige Resultate bezüglich der ersten drei oben erwähnten Gruppen von endosubjektiven Prädikaten besprechen: Prädikate des epistemischen Urteils, Prädikate der deontisch/alethischen Modalität sowie Scheinen-Prädikate.

\section{NATIVE SPEAKER-TESTS UND VORLÄUFIGE RESULTATE}

Als Korpus haben wir eine lange Reihe an Beispielen für die einzelnen Prädikate mit Hilfe von Google beschafft, für jedes Prädikat mit den folgenden Ergänzungen:

- $\quad$ klitisches (unbetontes) Dativpronomen: mi è chiaro ["mir ist klar"]

- Präposition $a$ + betontes Pronomen: a me è chiaro ["mir ist klar"]

- $\quad$ Präposition $a+$ Nominalphrase [+ menschlich]: è chiaro al bambino [“dem Kind ist klar"]

- $\quad$ Präposition $a+$ Nominalphrase [+ nicht-animiert]: è chiaro al mondo ["Der Welt ist klar"]

- Präposition per + betontes Pronomen: per lui è chiaro ["für ihn ist klar"]

- Präposition per + Nominalphrase [+ menschlich]: è chiaro per il mio interlocutore ["für meinen Gesprächspartner ist klar"]

- Präposition per + Nominalphrase [+ nicht-animiert]: è chiaro per il marxismo ["für den Marxismus ist klar"]

Es ist bekannt, dass ein Korpus von Google seine Nachteile (wie etwa inhomogene und teilweise hybride Textgenera und Register; Normverstöße ${ }^{3}$ ), aber auch seine Vorteile hat, da genau diese Abweichungen von der präskriptiven Norm Anlass zu Diskussionen mit den muttersprachlichen Informanten geben und dabei zu neuen Erkenntnissen führen können. Absolut ungrammatische Konstruktionen findet man wohl kaum in Google, man sei 


\section{ALEXANDRA KRATSCHMER}

jedoch auf der Hut vor Texten, welche von Nicht-native speakers verfasst oder sogar automatübersetzt sind.

Wir haben bis auf weiteres folgende Prädikate des epistemischen Urteils untersucht:

(9) è indubbio

'es ist zweifelsfrei'

(10) è certo

'es ist gewiss'

(11) è evidente

'es ist deutlich/evident'

(12) è chiaro

'es ist klar'

Darüber hinaus haben wir folgende Prädikate der deontisk/alethischen Modalität untersucht:

(13) occorre

'es ist vonnöten/notwendig'

(14) bisogna

'es ist vonnöten/notwendig'

(15) necessita

'es ist vonnöten/notwendig'

(16) basta

'es genügt/es ist hinreichend'

(17) è necessario

'es ist vonnöten/notwendig' 


\section{DER VERFLIXTE ITALIENISCHE DATIV - EIN (ERSTER) SEMANTISCH-GRAMMATIKALISCHER \\ ZÄHMUNGSVERSUCH}

\section{(18) è obbligatorio}

'es ist verpflichtend'

In Bezug auf die Scheinen-Prädikate können wir auf bereits existierende Untersuchungen verweisen (vgl. Kratschmer 2005a und 2005b).

Wir wollen im Anschluss unsere Resultate bezüglich è evidente und è chiaro aus dem epistemischen Bereich, bezüglich occorre, bisogna, necessita, è necessario, è obbligatorio und basta aus dem deontisch/alethischen Bereich sowie bezüglich der Scheinen-Prädikate sembrare und parere vorstellen.

\subsection{Prädikate des epistemischen Urteils}

Betreffs è evidente und è chiaro haben die konsultierten Muttersprachler einen Lesartunterschied zwischen diesen Prädikaten mit Ergänzungen auf der Basis von $a$ vs. per (für è evidente) und zwischen jenen mit dativischen Pronomen und $a$ Präpositionalphrasen auf der einen Seite und per-Präpositionalphrasen auf der anderen Seite (für è chiaro) festgestellt:

\begin{tabular}{|l|l|l|}
\hline \multicolumn{2}{|c|}{ "WISSEN" } & \multicolumn{1}{|c|}{ "ÜBERZEUGT SEIN" } \\
\hline $\begin{array}{l}\text { (19) ?a me è evidente } \\
\text { (20) È evidente a tutti }\end{array}$ & (21) Per me è evidente \\
\hline $\begin{array}{l}\text { (22) Mi è chiaro } \\
\text { (23) Gli è chiaro }\end{array}$ & (24) A lui è chiaro & (25) Per lui è chiaro \\
\hline
\end{tabular}

Die resultierende, kombinierte Lesart ist entweder "wissen" ("sapere") oder "überzeugt sein" ("essere convinti”), wie man an folgenden Beispielen und deren Übersetzungen sehen kann:

(19) ?Ecco perché a me è evidente [= io so] che l'ammontare dell'Irpef viene alla fine fatto pagare ai clienti finali. [http://www.ilgiornale.it/a.pic1?ID=40225]

'Deswegen ist (es) mir deutlich [= weiß ich], dass die Anhebung der Umsatz-/Mehrwertsteuer letzten Endes auf die Endabnehmer abgewälzt werden wird.' 
$(20)+(21)$

E' evidente a tutti [= tutti sanno], però, che qualche passo avanti deve essere compiuto e per me è evidente [= sono convinto] che va costruita una alternativa e strumenti che siano in grado di praticarla. [http://www.forumterzosettore. it/newsletter/12/12b.htm]

'Es ist jedoch allen klar [= alle wissen], dass irgendein Schritt vorwärts gemacht werden muss, und für mich ist klar [= ich bin überzeugt], dass eine Alternative geschaffen werden muss und Instrumente, welche diese praktizieren können.'

Alle muttersprachlichen Informanten haben im Übrigen è evidente "es ist deutlich" in Kombination mit Dativpronomen mi/gli ("mir/ihm") als nicht akzeptabel klassifiziert (Beispiele dafür gab es jedoch in Google). Geteilt waren die Meinungen bezüglich der Akzeptabilität von è evidente mit Präposition $a+$ betontem persönlichem Pronomen, a me ("mir", wie in Beispiel (19) aus Google). Die Kombination mit $a$ + Indefinitpronomen tutti "alle" wie in Beispiel (20) wurde einstimmig als normkonform empfunden.

$(24)+(22)$

"Senti, se tu hai problemi con la tua omosessualità non devi proiettarli su di me! Questo è il mio lavoro, mi capita ben di peggio, tipo toccare una modella idiota e appiccicosa che prende quel che faccio per delle avanches [sic] e non mi si stacca più di dosso!! Non ho alcun problema a toccare un uomo, almeno finché a lui è chiaro $[=$ lui sa] che io sto lavorando!" "Beh, sappi che mi è perfettamente chiaro! [= sappi che lo so perfettamente!]" [http://www. sheerajay.com/SJ_archive/racconti/PmS13.htm]

“"Hör mal, wenn du ein Problem mit deiner Homosexualität hast, dann projizier' sie nicht auf mich! Das hier ist meine Arbeit, mir passieren echt schlimmere Sachen, so wie ein idiotisches und aufdringliches Model anzufassen, die dann meine Gesten als Annäherungen auffasst und von mir nicht mehr runterkriecht!! Ich habe kein Problem damit, einen Mann anzufassen, zumindest solange es ihm klar ist [= er weiß], dass ich arbeite." "OK, versteh', dass mir das total klar ist [= dass ich das absolut weiß]!"'

(23) Balak [re di Moab] vuole sapere qual è il segreto di Israele poiché gli è chiaro [= lui sa] che una piccola nazione non può sopraffare dei giganti dell'epoca come Sichon e Og senza una qualche arma segreta. [http://digilander.libero.it/ parasha/archivio\%2058/5837.htm]

'Balak [König von Moab] möchte wissen, was Israels Geheimnis ist, denn ihm ist klar [= er weiß], dass eine kleine Nation ohne irgendeine Geheimwaffe keine zeitgenössischen Giganten wie Sichon und Og überwältigen kann. [Thora-Episode]' 


\section{DER VERFLIXTE ITALIENISCHE DATIV - EIN (ERSTER) SEMANTISCH-GRAMMATIKALISCHER ZÄHMUNGSVERSUCH}

Die Beispiele (24) und (22) illustrieren übrigens eine weitere Opposition: die Opposition zwischen dem wissenden und nicht-thematisierten Subjekt (22) (mi è chiaro) und dem wissenden und thematisieren Subjekt (24) (a lui è chiaro). Diese Opposition existiert im pronominalen Bereich, wo sowohl klitische, unbetonte Pronomen als auch von der Präposition $a$ abhängige betonte Pronomen zur Verfügung stehen, und kann dazu eingesetzt werden, die Informationsstruktur des Textes zu markieren: $a$ + betontes Pronomen markiert das thematisierte wissende Subjekt. Dahingegen ändert sich die Lesart des gesamten Prädikates im folgenden Beispiel (25), wo per das überzeugte Subjekt einleitet:

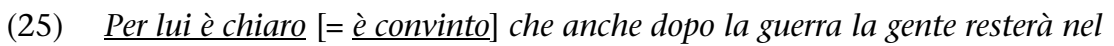
campo. [http://www.medicisenzafrontiere.it/msfinforma/lettere/ afghanstories_faiz.shtml]

'Für ihn ist klar [= er ist überzeugt], dass die Leute auch nach dem Krieg im Lager bleiben werden.'

Da die dativischen Ergänzungen einen Einfluss auf die Lesart haben, haben diese Ergänzungen möglicherweise auch unterschiedliche semantische Rollen in Bezug auf die unterschiedlichen resultierenden Prädikate, was wir in Abschnitt 6. untersuchen wollen.

\subsection{Prädikate, welche deontisch/alethische Modalität ausdrücken}

Im deontisch/alethischen Bereich haben die muttersprachlichen Informanten ebenfalls einen Unterschied zwischen auf der einen Seite Konstruktionen mit dativischem Pronomen und $a$-Präpositionalphrase und auf der anderen Seite perPräpositionalphrase festgestellt. Der Unterschied liegt jedoch nicht in der Lesart des Prädikats selbst, sondern in der Präsentation des (dativischen) modalen Subjekts: Konstruktionen mit dativischem Pronomen oder $a$-Präpositionalphrase installieren das genötigte Subjekt absolut, an sich, im Übrigen parallel mit einer Installation des genötigten Subjekts in Form eines grammatikalischen (nominativischen) Subjekts in der Paraphrase (io) sono costretto ("ich bin genötigt" ${ }^{4}$. Konstruktionen mit per-Präpositionalphrase heben dahingegen das genötigte Subjekt emphatisch ${ }^{5}$ hervor, das heißt, dass sie das genötigte Subjekt in ein Paradigma stellen, wo dieses einen Gegensatz in Bezug auf eine Reihe anderer möglicher, impliziter Kandidaten für dessen semantische Rolle aufbaut: io sono costretto ("ich [mit emphatischer Betonung] bin genötigt" oder, explizitiert: "es gibt jemanden (darunter mich), der genötigt ist"). 
AleXANDRA Kratschmer

\begin{tabular}{|c|c|c|}
\hline \multicolumn{2}{|c|}{$\begin{array}{l}\text { "GENÖTIGT SEIN" } \\
\text { (absolut) }\end{array}$} & \multirow{2}{*}{$\begin{array}{l}\text { "GENÖTIGT SEIN" } \\
\text { (paradigmatisiert) } \\
\text { (29) Per lui occorre }\end{array}$} \\
\hline (26) Mi occorre & $\begin{array}{l}\text { (27) A lui occorre } \\
\text { (28) Occorre a un ragazzo }\end{array}$ & \\
\hline (30) ?Gli bisogna & $\begin{array}{l}\text { (31) ?a lui bisogna } \\
\text { (32) ?bisogna a uno } \\
\text { scrittore }\end{array}$ & $\begin{array}{l}\text { keine authentischen } \\
\text { Beispiele im Korpus } \\
\text { (33) ?per Ovidio bisogna }\end{array}$ \\
\hline (34) Mi necessita & (35) Necessita al neonato & $\begin{array}{l}\text { (36) non necessita per i } \\
\text { nuovi clienti }\end{array}$ \\
\hline (37) Mi è necessario & $\begin{array}{l}\text { (38) È necessario a me/te } \\
\text { (39) È necessario al mailing } \\
\text { list manager }\end{array}$ & $\begin{array}{l}\text { (40) Per lui è necessario } \\
\text { (41) È necessario per il } \\
\text { programmatore }\end{array}$ \\
\hline \multirow[t]{2}{*}{ (42) Mi è obbligatorio ${ }^{6}$} & $\begin{array}{l}\text { keine authentischen } \\
\text { Beispiele im Korpus } \\
\text { (43) a me è obbligatorio }\end{array}$ & (44) Per te è obbligatorio \\
\hline & $<<<<<<$ & $\begin{array}{l}\text { (45) È obbligatorio per il } \\
\text { cittadino italiano }\end{array}$ \\
\hline \multicolumn{2}{|c|}{$\begin{array}{c}\text { "BLOSS GENÖTIGT SEIN"7 } \\
\text { (absolut) }\end{array}$} & $\begin{array}{l}\text { "BLOSS GENÖTIGT } \\
\text { SEIN" } \\
\text { (paradigmatisiert) }\end{array}$ \\
\hline (46) Mi basta & $\begin{array}{l}\text { (47) A me basta } \\
\text { (48) Basta a un uomo }\end{array}$ & (49) Per lui basta \\
\hline
\end{tabular}

Wir bringen nun Beispiele für diese Konstruktionen (inklusive Anmerkungen bezüglich einiger ihrer Besonderheiten): 


\section{DER VERFLIXTE ITALIENISCHE DATIV - EIN (ERSTER) SEMANTISCH-GRAMMATIKALISCHER ZÄHMUNGSVERSUCH}

(26) Mi occorre che mi diciate cortesemente dove trovare un tool di diagnostica/ riparazione/ecc. [http://www.club.cdfreaks.com/archive/topic/42589.html]

'Mir ist vonnöten, dass ihr mir freundlicherweise sagt, wo man ein Diagnose-/Reparatur/etc.-tool finden kann.'

(27) L'uomo possiede l'istinto degli animali, quello che gli necessita lo cerca. Prima di cadere in uno stato di prostrazione lo spirito di conservazione lo spinge a ricercare cosa a lui occorre per assicurargli l'integrità della sua salute. [http://www.ricetteonline.com/tipi_cucina/cucina-vegetariana/chi-troppochi-niente-php.php]

'Der Mensch besitzt den Instinkt der Tiere, was er braucht, sucht er. Bevor er in einen Zustand der Schwächung verfällt, treibt ihn der Erhaltungstrieb dazu, das zu suchen, was ihm zur Erhaltung seiner Gesundheit vonnöten ist.'

(28) perché nessun alimento contiene contemporaneamente tutto ciò che occorre a un ragazzo per l'accrescimento. [http://www.it.health.yahoo.net/c_special.asp? $\mathrm{id}=10032 \& \mathrm{~s}=1 \& \mathrm{c}=7]$

'da kein Nahrungsmittel gleichzeitig all das enthält, was einem jungen Menschen zum Wachstum vonnöten ist.'

(29) Ma De Rosa è malato e bisognoso di cure. Per lui occorre $[=\underline{\text { lui }}$ [mit emphatiscer Betonung] è costretto a ....] anche un tutore perché ha perso la capacità giuridica autonoma: [...]. [http://www.associazioneantigone.it/ rassegnastampa/ ottobre/13-19\%20ottobre2003-2.htm]

'Aber De Rosa ist krank und pflegebedürftig. Für ihn ist auch ein Vormund vonnöten, da er seine juristische Mündigkeit verloren hat: [...].'

Bezüglich bisogna ${ }^{8}$ muss erwähnt werden, dass ein deutlicher generationsbedingter Unterschied hinsichtlich der Akzeptabilität dieses Lexems in Zusammenhang mit dativischen Ergänzungen festgestellt werden konnte. Je nachdem, ob die Informanten vor oder nach dem Zweiten Weltkrieg geborenen waren, wurden die in Google durchaus belegten Konstruktionen entweder als gängig oder als archaisch und nicht länger normkonform beurteilt. Die Informanten verwiesen dagegen auf die äußerst gebräuchliche Konstruktion avere bisogno di "Bedarf an ... haben" im Hinblick auf eine moderne Entsprechung. Dies galt sowohl für die Beispiele (30) und (31) von Google als auch für unser eigens konstruiertes Beispiel (32). Nachdem wir sodann eine große Menge an Beispielen aus Google mit bisogna per il/la/i/le "es ist notwendig für + best. Art. m./f. sg./pl." untersucht hatten, ohne auf relevante Beispiele zu treffen, änderten wir Beispiel (31) in eine Konstruktion mit perPräpositionalphrase mit nominalem Regimen (33). Die Reaktion war Akzeptanz von der älteren und Beurteilung als veraltet von der etwas jüngeren Generation. 
(30) ?ognuno raccoglierà quanto gli bisogna per il suo sostentamento. [http://www. namir.it/JOHANN/1babilon.htm]

'jeder wird so viel sammeln, wie er für seinen Unterhalt braucht.'

(31) ?così che a lui $[=\underline{\text { a Ovidio }]}$ bisogna una pagina per farci veder quello che Dante ci fa vedere in una terzina [Leopardi]. [http://www.leopardi.it/zibaldone1. php]

'so, dass ihm [= Ovid] eine Seite vonnöten ist, um uns zu zeigen, was Dante uns in einer Terzine [= einem Dreizeiler] zeigt.'

(32) ?quello che bisogna a uno scrittore non bisogna sempre a un giornalista

'was einem Autor vonnöten ist, ist nicht immer einem Journalisten vonnöten'

(33) ?per Ovidio bisogna [= Ovidio [mit emphatischer Betonung] è costretto a ...] una pagina per farci veder quello che Dante ci fa vedere in una terzina

'für Ovid ist eine Seite vonnöten, um uns zu zeigen, was Dante uns in einer Terzine zeigt'

(34) Abito a Milano, vorrei ristrutturare il bagno, cambiare le tubazioni vecchie e sostituire sanitari e piastrelle, oltre all'impresa e ad un idraulico per eseguire $i$ lavori sono obbligato a fare delle pratiche in comune e quindi mi necessita l'assistenza di un professionista? [http://www.forum.architetto.info/ viewtopic.php?p=321\&sid=3f06f0568f41a897665d06e51ee5a657]

'Ich wohne in Mailand, ich möchte mein Bad renovieren, alte Rohre austauschen und Sanitäreinrichtung und Fliesen ersetzen, abgesehen von der Firma und dem Installateur, welche die Arbeiten ausführen sollen, bin ich verpflichtet, bei der Gemeinde die Papiere zu erledigen, ist mir daher die Hilfe eines Fachmannes vonnöten?'

(35) Capiente borsa per trasportare tutto quello che necessita al neonato [http:// www.shopping.kelkoo.it/b/a/ss_foppapedretti_borsa_necessaire.html]

'Geräumige Tasche um alles zu transportieren, was dem Neugeborenen vonnöten ist'

(36) Non necessita per $i$ nuovi clienti $[=$ I nuovi clienti [mit emphatischer Betonung] non sono costretti a ...] nessun deposito cauzionale né fideiussione bancarie [sic] o assicurative [sic], anzi è possibile recuperare (con apposita richiesta scritta) il deposito cauzionale a suo tempo versato all'Enel. [http:// www.firenze.cna.it/FTPimmaginiCNA/Convenzioni/GuidaVantaggi2005.p df]

'Für die neuen Kunden ist keine Kaution oder Bank- oder Versicherungsgarantie vonnöten, es ist im Gegenteil möglich (auf schriftliches Ansuchen) die Kaution ausbezahlt zu bekommen, welche seinerzeit der ENEL $[=$ der italienischen staatlichen Elektrizitätsgesellschaft] gestellt wurde.'

(37) Vivo sola e spesso mi è necessario congelare il cibo [http://www.ciao.it/sspElettrodomestici_168360.html]

'Ich lebe alleine und oft ist es mir daher notwendig, Essen einzufrieren' 


\section{DER VERFLIXTE ITALIENISCHE DATIV - EIN (ERSTER) SEMANTISCH-GRAMMATIKALISCHER ZÄHMUNGSVERSUCH}

(38) La generosità non consiste nel donare a me ciò che è necessario a me più che a te, ma nel donare a me quel che è necessario più a te che a me. [http://www. giuseppecirigliano.it/Aforismi\%20e\%20citazioni\%20(3).htm]

'Großzügigkeit besteht nicht darin, mir das $\mathrm{zu}$ geben, was mir notwendiger ist als dir, sondern mir das zu geben, was dir notwendiger ist als mir.'

Die letzten beiden Beispiele illustrieren im Übrigen, genauso wie bei den epistemischen Prädikaten, die Opposition zwischen dem nicht-thematisierten (genötigten) Subjekt (37) und dem thematisierten (genötigten) Subjekt (38), beide in ihrer absoluten, nicht emphatischen Lesart. Thematisierung (mittels $a$ Präpositionalphrase) und Fokalisierung (mittels per-Präpositionalphrase) müssen voneinander unterschieden werden: die Thematisierung kann zwar - wie in (38) - auch einen Gegensatz installieren, der Gegensatz besteht allerdings zwischen konkreten Größen, welche im (Kon-)Text vorhanden sind und über welche abwechselnd gesprochen wird ("mir" vs. "dir": eine Frage der Informationsstruktur), und nicht (wie bei der Fokalisierung) zwischen einer bestimmten Größe und einem (impliziten) Paradigma mit einer unbekannten Anzahl an Mitgliedern, wie in (40) und (41) unten. Der Ausdruck è necessario $\underline{a}$

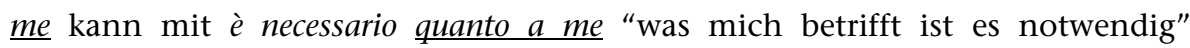
paraphrasiert werden: quanto a me kann als Paraphrasierungstest verwendet werden, um den Status eines sprachlichen Elements als thematisiert festzustellen.

(39) Tale account è necessario al mailing list manager per assicurare la gestione e la configurazione delle liste di distribuzione [http://www.ecomstation.it/pido2/ home/djspider8/major.html]

'Ein derartiges Konto ist dem mailing list manager notwendig, um die Verwaltung und die Erstellung der Verteilerlisten sichern zu können'

(40) Il prete vive di peccati, per lui è necessario [= lui [mit emphatischer Betonung] è costretto a ....] che si pecchi. [http://www.lgxserver.uniba.it/lei/rassegna/ 000825e.htm]

'Der Priester lebt von den Sünden, für ihn ist es notwendig, dass man sündigt.'

(41) Il materiale seguente è necessario per il programmatore [= il programmatore [mit emphatischer Betonung] è costretto a ...] [http://www.tldp.org/ linuxfocus/Italiano/November2004/article352.shtml]

'Das folgende Material ist notwendig für den Programmierer' 
Das Prädikat ̀̀ obbligatorio "es ist verpflichtend" weist einige besondere Eigenschaften auf, welche an jene erinnern, welche wir in Abschnitt 2. bezüglich è (im)possibile "es ist (un)möglich" angeführt haben: es kann nicht mit $a$ Präpositionalphrasen mit nominalem Regimen kombiniert werden ( ${ }^{\star} \grave{e}$ obbligatorio al cittadino italiano "es ist ist verpflichtend für den italienischen Staatsbürger"). Die einzig mögliche Form ist hier eine per-Präpositionalphrase wie im Beispiel (45) unten. Damit fällt die Opposition zwischen $a$ - und perFormen weg und die per-Formen verlieren ihre emphatische Lesart: (45) präsentiert sein genötigtes Subjekt absolut, ganz parallel zu (42), wo das klitische Pronomen die gleiche Funktion innehat. Dahingegen findet man in pronominalen Bereich, wo sowohl $a$ - als auch per-Präpositionalphrasen (und klitische Pronomen) möglich sind, die bekannte Opposition zwischen dem absoluten $(42) /(43)^{10}$ und dem paradigmatisierten, emphatischen genötigten Subjekt (44).

(42) In quanto cittadino italiano, sin dall'età di 14 anni mi è obbligatorio portare sempre con me un valido. [sic] documento d'identità (o passaporto). [http:// www.communicationvalley.it/cryptogramPdf/Maggio2004.pdf]

'Als italienischer Staatsbürger ist es mir ab dem vollendeten 14. Lebensjahr verpflichtend, stets einen gültigen Personalausweis (oder Pass) bei mir zu tragen.'

(43) A me è obbligatorio, a te no. ${ }^{11}$

'Für mich ist es verpflichtend, für dich nicht.'

(44) per te è obbligatorio $[=\underline{t u}$ [mit emphatischer Betonung] sei costretto a ...] prendere un Hd grosso. [http://www.freeforumzone.com/viewmessaggi. aspx?f=2440\&idd=6825\&p=2]

'für dich ist es verpflichtend, eine große Festplatte zu wählen.'

(45) È obbligatorio per il cittadino italiano denunciare al commissariato di Polizia di zona l'ospitalità data al cittadino straniero. [http://www.spazioinwind. libero.it/ik1zyw/other/laurea.html]

'Es ist verpflichtend für den italienischen Staatsbürger, bei der lokalen Polizeistation zu melden, wenn man einen ausländischen Bürger beherbergt.'

(46) Volerei tranquillamente, mi basta che l'aereo sia collegato a terra da un palo lunghissimo che lo sostiene sempre. [http://www.it.health.yahoo.net/ c_special.asp? $\mathrm{id}=11184 \& \mathrm{~s}=1 \& \mathrm{c}=14]$

'Ich würde ganz beruhigt fliegen, mir genügt es, dass das Flugzeug mittels einer sehr langen Stange an der Erde befestigt ist, welche es die ganze Zeit trägt.' 


\section{DER VERFLIXTE ITALIENISCHE DATIV - EIN (ERSTER) SEMANTISCH-GRAMMATIKALISCHER ZÄHMUNGSVERSUCH}

(47) A me basta che lei dica il nome di Pinocchio e mi viene un sommovimento dell'anima, un'esuberanza. [http://www.raiuno.rai.it/popup_articolo/ 0,8070,125\%5E1917,00.html]

'Mir genügt, dass sie den Namen Pinocchio ausspricht, und ich verspüre einen Aufruhr in der Seele, eine Überschwenglichkeit.'

(48) Si preferisce impiegare un uomo perché l'uomo è una macchina che obbedisce alla voce e perché basta a un uomo ricevere un ordine [http://www. nextonline.it/archivio/15/17.htm]

'Man zieht es vor, einen Mann anzustellen, weil der Mann eine Maschine ist, welche aufs Wort hört und weil es einem Mann genügt, einen Befehl zu empfangen'

(49) Purtroppo Google non riesce a valutare la qualità della roba che indicizza, per

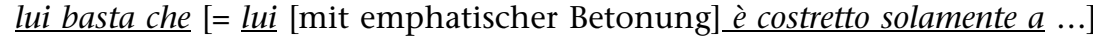
sia linkata da molte altre pagine. [http://blogs.devleap.com/marco/archive/ 2005/04/19/3296.aspx]

'Leider kann Google nicht die Qualität des Zeugs beurteilen, welches es indexikalisiert, für es [= für Google] genügt es, dass es Links von vielen anderen Seiten hat.'

\subsection{Scheinen-Prädikate}

Wir bringen an dieser Stelle eine Zusammenfassung unserer Resultate bezüglich einiger für uns hier relevanter ${ }^{12}$ semantischer und grammatikalischer Gegebenheiten der beiden italienischen Scheinen-Prädikate sembrare und parere ${ }^{13}$, wie wir sie in Kratschmer (2005a) bzw. (2005b) ausführlicher diskutiert haben. $\mathrm{Zu}$ den semantischen Eigenschaften, welche uns interessieren, gehören epistemische Beurteilung und Evidentialität (Angaben bezüglich der Quelle und/oder der Art und Weise der Beschaffung einer Informationseinheit), zu den grammatikalischen die Kompatibilität mit dativischen Ergänzungen. Die Prädikate sembrare und parere treten in drei unterschiedlichen Konstruktionen auf: einerseits unpersönlich mit konjunktivischem Kompletivsatz, andererseits mit persönlichem, Verbalkongruenz auslösendem Subjekt, hier entweder mit untergeordnetem Infinitiv (raising) oder ohne jegliches untergeordnete Verb (small clause).

Die Kompletivkonstruktion sembra/pare che ("es scheint, dass") hat drei Lesarten, welche sich in unterschiedlicher Kompatibilität mit anderen Satzelementen widerspiegeln. Es handelt sich dabei zunächst um die Wiedergabe eines Gerüchts (50), wobei in dieser Lesart u.a. die dativische Ergänzung, welche das modale Subjekt ausdrücken würde ( $m i$, "mir"), nicht zugelassen ist. Die Kompatibilität 
mit der vom Gerücht abweichenden Meinung des Senders macht die vom Sender verschiedene Quelle des vehikulierten Sachverhaltes deutlich:

(50) ( $\left.{ }^{*} \mathrm{Mi}\right)$ Sembra/pare che Gianni sia malato. Ma, a dire la verità, sono sicuro che non è così.

'Es scheint $\left({ }^{*} \mathrm{mir}\right)$, dass Gianni krank ist $\cong$ Es heißt, Gianni sei krank/Gianni soll krank sein. Aber ehrlich gesagt bin ich davon überzeugt, dass das nicht stimmt.'

Daneben kann dieselbe Konstruktion eine eigene Inferenz auf der Basis fremder Indizien ausdrücken (51), eine Lesart, welche ebenfalls mit einem dativischen modalen Subjekt inkompatibel ist:

(51) “( “Mi) Sembra/pare che Gianni sia malato." "Perché?" "Perché Maria dice che è pallido e Francesco dice che non esce più."

"'Es scheint (*mir), dass Gianni krank ist." "Warum (denn das)?" "Weil Maria sagt, dass er bleich ist, und Francesco sagt, dass er nicht mehr ausgeht."

Zum Schluss kann sembra/pare che auch noch eine eigene Inferenz auf der Basis eigener Indizien markieren (52), wo schließlich ein dativisches modales Subjekt zugelassen ist:

(52) “(Mi) Sembra/pare che Gianni sia malato." "Perché?" "Perché è pallido e non esce più."

“"Es scheint (mir), dass Gianni krank ist $\cong$ Mir scheint wirklich/Es kommt mir wirklich vor, dass Gianni krank ist." "Warum (denn das)?" "Weil er bleich ist und nicht mehr ausgeht."

Raising-Konstruktionen ${ }^{14}$ drücken stets Inferenzen aus, wobei Unterschiede bezüglich der untergeordneten Prädikation zu verzeichnen sind. Bezeichnet die untergeordnete Prädikation einen Sachverhalt, der für den Sender nicht direkt perzeptiv zugänglich ist, dann ist der dativische Modusträger nicht zugelassen. Dies gilt sowohl für Kontexte, wo sich der Sender auf eigene (53) als auch fremde Indizien stützt, um zu seiner Beurteilung zu gelangen (54).

"Il colpevole ("mi) sembra/pare essere una donna." "Perché?" "Perché il patologo dice che le ferite sono state inflitte con poca forza."

“"Der Täter scheint ("mir) eine Frau zu sein." "Warum (denn das)?" "Der Pathologe sagt, dass die Wunden mit geringer Kraft zugefügt wurden."” 


\section{DER VERFLIXTE ITALIENISCHE DATIV - EIN (ERSTER) SEMANTISCH-GRAMMATIKALISCHER ZÄHMUNGSVERSUCH}

(54) "Il colpevole (*mi) sembra/pare essere una donna." "Perché?" "Perché ho sentito un profumo femminile sul luogo del reato."

“"Der Täter scheint (*mir) eine Frau zu sein." "Warum (denn das)?" "Ich habe Damenparfum am Tatort gerochen."

Drückt die untergeordnete Prädikation hingegen einen Sachverhalt aus, der dem Sender sehr wohl direkt perzeptiv zugänglich ist, dann ist die Konstruktion mit einem dativischen modalen Subjekt kompatibel (55). Dabei ist zu beachten, dass die Raising-Konstruktion trotz der perzeptiven Zugänglichkeit des Prädikates eine Inferenz ausdrückt. Die der Inferenz zugrunde liegenden Indizien werden gleichsam zu einem Gesamtbild, einem Mosaik zusammengesetzt. Der Charakter der Inferenz ist hier daher eher additiv, wohingegen er in (53) und (54) rein abduktiv ist.

(55) Una porticina, in lamiera sommariamente verniciata di bianco, (le) sembrava essere l'unica via d'uscita. ${ }^{15}$

'Eine kleine Tür, aus sehr oberflächlich weiß getünchtem Blech, schien (ihr) der einzige Weg hinaus zu sein.' (Grasso 2003: 21)

Small clause-Konstruktionen ${ }^{16}$ drücken stets eine unmittelbare Bewertung eines Sachverhaltes aus, welche je nach untergeordneter Prädikation eine perzeptive (56) oder eine intellektuelle Bewertung (57) ist. Diese Konstruktionen sind stets mit dativischen modalen Subjekten kompatibel.

(56) Gianni (mi) sembra/pare pallido.

'Gianni scheint (mir) bleich [zu sein] $\cong$ kommt mir bleich vor.'

Quest'idea (mi) sembra/pare nuova.

'Diese Idee scheint mir neu [zu sein] $\cong$ kommt mir neu vor.'

Allen Konstruktionen ist gemeinsam, dass sie zusätzlich zu den einzelnen evidentiellen Nuancen eine epistemische Beurteilung ausdrücken, welche stets die gleiche ist, namentlich einen Vorbehalt, welcher mit "vorläufige Konklusion" etikettiert werden kann. Die "vorläufige Konklusion" versteht sich als epistemische Nuancierung des in der untergeordneten Prädikation ausgedrückten Sachverhaltes p, als ein - womöglich nicht endgültiger - Schritt auf dem kognitiven Prozess der Beurteilung von p: "auf der Basis der im Augenblick zur Verfügung stehenden Informationen kann $\mathrm{p}$ eine hohe 
Wahrscheinlichkeit zugesprochen werden; es kann jedoch nicht ausgeschlossen werden, dass $\mathrm{p}$ im Anschluss an das Auftauchen neuer Informationen revidiert werden muss".

Ebenso für alle sembrare- und parere-Konstruktionen gilt, dass sie, sofern sie dativische Ergänzungen zulassen, diese nur in Form von echten dativischen klitischen Pronomen (mi, ti, gli etc. "mir", "dir", "ihm") oder in Form von mit a eingeleiteten Präpositionalphrasen (a me "mir", all'insegnante "dem Lehrer") zulassen. Mit per eingeleitete Präpositionalphrasen sind absolut ausgeschlossen.

\section{EINFÜHRUNG IN DIE POLYPHONIETHEORIE: SCAPOLINE}

Da wir im Anschluss die Problemstellung der dativischen Ergänzungen bei den endosubjektiven Prädikaten des Italienischen aus polyphonietheoretischer Sicht beleuchten wollen, ist es an dieser Stelle vonnöten, eine kurze Einführung in den von uns gewählten Theorieapparat voranzustellen. Wir arbeiten im Rahmen von ScaPoLine, der "Théorie Scandinave de la Polyphonie Linguistique" gemäß Nølke/Fløttum/Norén (2004).

ScaPoLine wurde in einem mehrjährigen, von Nordiska samarbetsnämnden för humanistisk och samhällsvetenskaplig forskning (NOS-HS) ${ }^{17}$ geförderten panskandinavischen Forschungsprojekt vom Dänen Henning Nølke (Aarhus Universitet), der Norwegerin Kersti Fløttum (Universitet i Bergen) und der Schwedin Coco Norén (Uppsala Universitet) entwickelt und hat sich im Anschluß daran besonders im französischsprachigen Raum große Anerkennung geschaffen.

ScaPoLine wurde namentlich auf Französisch entwickelt (die beteiligten Forscher sind alle Französisten) und die ursprüngliche Terminologie entstammt daher einer französischsprachigen Tradition, genauer gesagt jener der Schule um Oswald Ducrot (vgl. z.B. Ducrot 1984). Sprogligt Polyfoninetvoerk ("Sprachliches Polyphonienetzwerk"), eine von 2003 bis 2006 mit Mitteln des dänischen Statens Humanistiske Forskningsråd ("Geisteswissenschaftlicher Forschungsrat des Staates Dänemark") geförderte Initiative von Rita Therkelsen (Roskilde Universitetscenter), hat es sich zum Ziel gesetzt, die Erkenntnisse der ScaPoLine nicht-französischkundigen Linguisten Skandinaviens zu vermitteln. Dies geschah zunächst durch Rita Therkelsens Übersetzung des zentralen Kapitels 2 


\section{DER VERFLIXTE ITALIENISCHE DATIV - EIN (ERSTER) SEMANTISCH-GRAMMATIKALISCHER ZÄHMUNGSVERSUCH}

("2. ScaPoLine") der Publikation Nølke/Fløttum/Norén (2004) und damit gleichzeitig auch der Terminologie ins Dänische. Mittlerweile wurde im Rahmen des Netzwerkes die Terminologie auch ins Englische (vgl. Nølke 2005a) und Deutsche (vgl. Kratschmer 2006) übersetzt. Die weiteren Resultate der Netzwerkarbeit sind auf der Homepage des Netzwerkes einsichtig: [http://babel.ruc.dk/polyfoni/].

Der polyphonietheoretische Ansatz ScaPoLine beschreibt das Vorhandensein sprachlicher Spuren von mehr als einem Gesprächsteilnehmer ("Mehrstimmigkeit") innerhalb von Äußerungen. ScaPoLine versteht sich als instruktionelle Semantik, das heißt, dass sie davon ausgeht, dass sprachliche Äußerungen Anweisungen $\mathrm{zu}$ ihrer Dekodifizierung und Interpretation vehikulieren. Diese Anweisungen beinhalten explizite und implizite Elemente. Manche Elemente werden als Variable verstanden, für welche der Empfänger im Rahmen der Interpretation konkrete Saturanten finden muss. Für ihre Beschreibung von Mehrstimmigkeit formalisiert ScaPoLine die Gesprächsteilnehmer als so genannte "Diskursindividuen" ("DIs"), welche als Quellen von "Standpunkten" (semantischen Inhalten) beschrieben werden und welche mit diesen Standpunkten in einem bestimmten Verhältnis (= "DSRelation") stehen. Genauer gesagt stellen die Quellen von Standpunkten Variable dar, welche durch konkrete Diskursindividuen gesättigt werden können, aber nicht müssen. So kann etwa ein Satz, welcher eine polemische Negation enthält (z.B. Dieses Buch ist nicht gut), analysiert werden in einen Standpunkt 1 "dieses Buch ist gut", dessen Quelle eine ungesättigte Variable darstellt (d.h., dass das entsprechende konkrete Diskursindividuum sprachlich nicht ausgedrückt ist ${ }^{18}$ ), und einen Standpunkt 2 "Standpunkt 1 ist falsch", als dessen Quelle in der Standardinterpretation der Sender einzusetzen ist. Das Verhältnis des Senders zu Standpunkt 1 ist jenes der Zurückweisung, während jenes zu Standpunkt 2 "Verantwortlichkeit" darstellt. ScaPoLine betrachtet die Diskursindividuen als Bilder von (reellen oder potentiellen, gedachten) Gesprächsteilnehmern - Bilder, welche durch den Sender in seiner Rolle als Textkonstrukteur ("LOC" ${ }^{19}$ ) geschaffen werden. Diese Bilder umfassen solche von dritten Personen (individuelle oder kollektive ${ }^{20}$ Personen), vom Empfänger/Gesprächspartner, aber auch vom Sender selbst. Was nun die Senderdiskursindividuen betrifft, unterscheidet ScaPoLine zwischen dem Senderbild, welches den Sender als Diskursindividuum mit Geschichte darstellt, 
als Wesen, welches seinen Standpunkt über längere Zeit erworben und beibehalten hat (S), und jenem Senderbild, welches den Sender als Diskursindividuum darstellt, welches nur in der aktuellen Äußerung existiert, wo es hic et nunc zu einem Standpunkt Stellung nimmt $\left(\mathrm{s}_{0}\right)$.

Eine so genannte "polyphone Konfiguration"²1 (das Zusammenspiel mehrerer Stimmen in einer Äußerung), in welcher nur Senderbilder vorkommen, wird "interne Polyphonie" genannt, eine Konfiguration, wo außer den Senderbildern noch andere Diskursindividuen aufscheinen, dagegen "externe Polyphonie".

\section{Die DATIVISCHEN SEMANTISCHEN ROLLEN IN DEN ENDOSUBJEKTIVEN PRÄDIKATEN: POLYPHONE ANALYSE}

Wir beginnen mit folgender Hypothese: die Ausdrücke in den in 4.1. und 4.2. präsentierten Gegensatzpaaren ("ich weiß" vs. "ich bin überzeugt"; "ich bin genötigt" vs. "es gibt jemanden (darunter mich), der genötigt ist") geben unterschiedliche Instruktionen in Bezug auf die polyphone Dekodierung. Sie instanziieren mit anderen Worten unterschiedliche polyphone Konfigurationen mit unterschiedlichen Diskursindividuen und/oder Standpunkten. Es ist eventuell möglich, eine bestimmte semantische Rolle einem bestimmten Diskursindividuum inklusive dessem Standpunkt zuzuschreiben. Stellt man die relevanten polyphonen Konfigurationen einander gegenüber, wird möglicherweise deutlich, welches Diskursindividuum die relevante semantische Rolle innehaben könnte.

\subsection{Prädikate, welche eine epistemische Beurteilung ausdrücken}

Um uns einer polyphonen Analyse annähern zu können, paraphrasieren wir zunächst die relevanten Konstruktionen so, dass deren modales Subjekt als grammatikalisches Subjekt steht:

$$
\begin{gathered}
\text { Das wissende Subjekt } \\
\text { Mi è chiaro ("mir ist klar") } \\
\cong \text { (io) so che ("ich weiß, dass") }
\end{gathered}
$$

Das überzeugte Subjekt Per me è chiaro "für mich ist klar" $\cong$ (io) sono convinto che ("ich bin überzeugt, dass")

Nun explizitieren wir die Bedeutung dieser Paraphrasen, wodurch deren polyphone Natur deutlich wird: 


\title{
DER VERFLIXTE ITALIENISCHE DATIV - EIN (ERSTER) SEMANTISCH-GRAMMATIKALISCHER ZÄHMUNGSVERSUCH
}

\author{
"Ich weiß, dass p" \\ "Ich bin überzeugt, dass p" \\ "es gibt bezüglich p eine objektiv "ich habe mir bezüglich p eine \\ zugängliche Wahrheit, eine Wahrheit, Meinung gebildet und diese lautet, \\ welche der Gemeinschaft zugänglich dass p zutrifft" \\ ist und welche gleichzeitig auch mir \\ zugänglich ist"
}

Diese Inhalte können im Rahmen der ScaPoLine nach Nølke/Fløttum/Norén (2004), folgendermaßen formalisiert werden:

"Ich weiß, dass p"

"Ich bin überzeugt, dass p"

MAN: wahr (p) [objektivierende

Instanz]

S: wahr (p)

S: wahr (p)

$\mathrm{s}_{0}:$ wahr (p)

$\mathrm{s}_{0}:$ wahr $(\mathrm{p})$

Der Unterschied liegt also im Einbeziehen der objektivierenden Instanz MAN: der Sender behauptet entweder, zu einer Wahrheit Zugang zu haben, für welche er die Gemeinschaft als Garanten einsetzt ("ich weiß, dass"), oder er beschränkt sich darauf, über seinen Meinungsbildungsprozesses zu informieren ("ich bin überzeugt, dass").

Will man nun etwas über die verschiedenen dativischen semantischen Rollen von $\mathrm{mi} / \mathrm{a}$ me vs. per me in Zusammenhang mit dem Prädikat è chiaro sagen und sucht man nach einem Diskursindividuum innerhalb der oben genannten polyphonen Konfigurationen, welche diese dativische semantische Rolle innehat, dann muss dies ein Diskursindividuum sein, welches koreferent mit dem kognitiven Subjekt ist (das heißt mit mi/a me/per me "mir" in der konkreten Konstruktion oder mit io "ich" in der Paraphrase io so/io sono convinto "ich weiß"/"ich bin überzeugt"). Die Wahl steht damit zwischen den Senderdiskursindividuen $S$ und $S_{0}$. $S$ und $S_{0}$ sind jedoch in beiden Konfigurationen mit den gleichen Standpunkten verbunden. Der Unterschied zwischen den beiden Konfigurationen liegt rein im Diskursindividuum MAN, welches jedoch nicht mit dem kognitiven Subjekt io koreferent ist. Daraus muss - etwas überraschend - geschlossen werden, dass der Unterschied bezüglich der semantischen Rolle von mi/a me vs. per me in Verbindung mit dem Prädikat è 
chiaro nicht mit einem einzelnen Diskursindividuum assoziiert werden kann: die semantische Rolle liefert vielmehr Interpretationsinstruktionen bezüglich einer gesamten polyphonen Konstellation.

\subsection{Prädikate, welche deontisch/alethische Modalität ausdrücken}

Auf die gleiche Art und Weise wie bei den epistemischen Prädikaten wollen wir mit der Paraphrasierung der relevanten Konstruktionen beginnen, welche deontisch/alethische Modalität ausdrücken, sodass das modale Subjekt als grammatikalisches Subjekt zu stehen kommt.

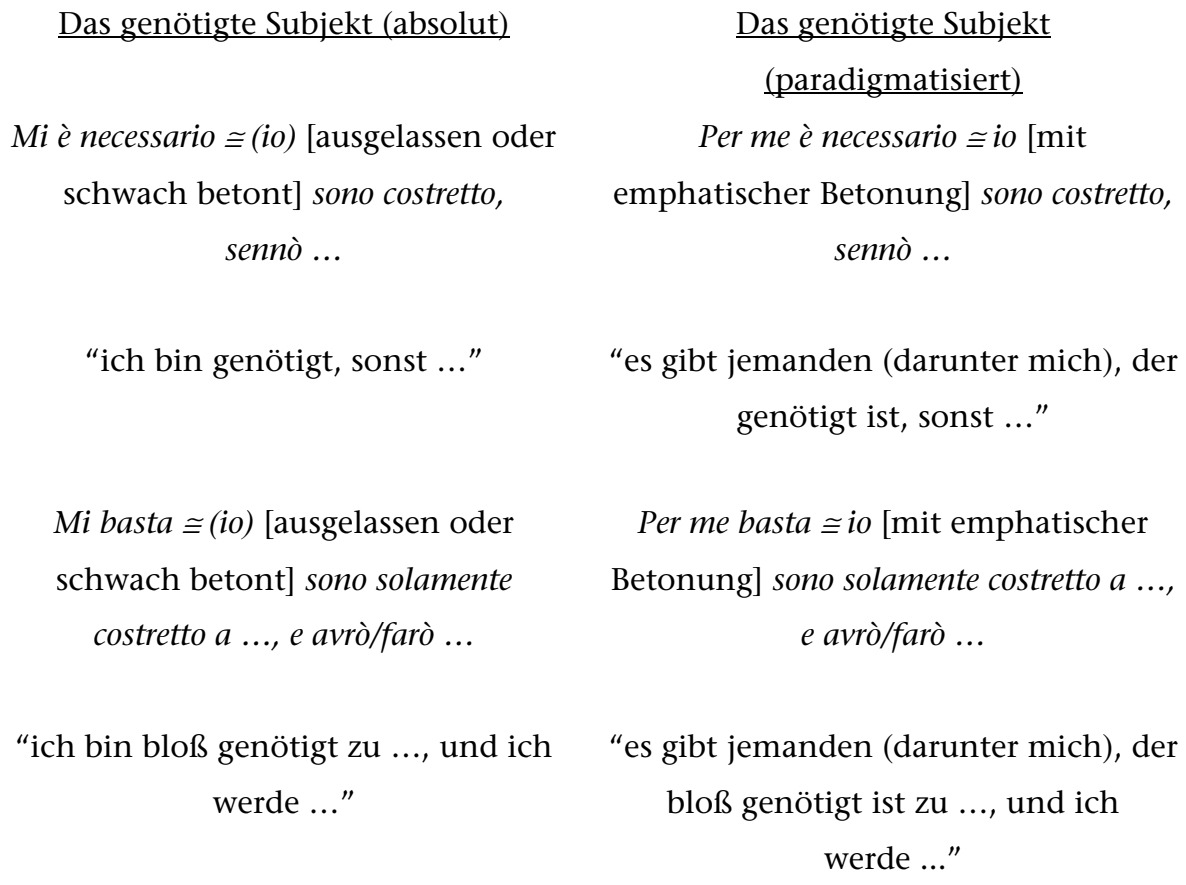

"es gibt jemanden (darunter mich), der bloß genötigt ist zu ..., und ich werde ..."

Wie man sehen kann, betrachten wir basta "es ist hinreichend" als eine Variante von è necessario "es ist notwendig". Genauer gesagt können diese beiden Prädikate als Raisonnements explizitiert werden, welche auf notwendigen (è necessario) oder hinreichenden (basta) Bedingungen basieren. Wie wir in Kratschmer (1998) und (2005c) gezeigt haben, können Notwendigkeit und "Hinreichendheit" als finale Raisonnements ausgedrückt werden, wo ein Sender folgende propositionelle Größen miteinander verbindet: einerseits den Ausdruck einer Gesetzmäßigkeit (= praemissa maior), welche ein Antezedens mit einem Konsequens verbindet $(\mathrm{p} \rightarrow \mathrm{q})$, andererseits den Wunsch des Senders, q zu 
erreichen (= praemissa minor), und schließlich, als Konklusion, den Wunsch des Senders, p zu erreichen (das heißt die Erfüllung der Bedingung, welche zu q führt). Wenn etwas notwendig/hinreichend ist, so ist es dies stets im Hinblick darauf, ein Ziel zu erreichen oder etwas Unerwünschtes zu umgehen, obwohl dies rein sprachlich oft implizit bleibt. Sobald man es unterlässt, sich selbst dem Wunsch, einen Sachverhalt zu erreichen oder zu umgehen, zu unterwerfen, fällt die Notwendigkeit weg (das heißt die Notwendigkeit, die Bedingung zu erfüllen, die zum Eintritt des Sachverhaltes führt oder diesen verhindert). Wir meinen darüber hinaus, dass zwischen Raisonnements, welche auf von Menschen geschaffenen Gesetzen (deontische Kontexte) aufbauen, und Raisonnements, welche auf Naturgesetzen aufbauen (alethische Kontexte), kein sprachrelevanter Unterschied besteht, weshalb wir diese alle unter dem selben Etikett "deontisch/alethisch" behandeln.

In semi-natürlicher Sprache explizitiert können diese deontisch/alethischen Kontexte folgendermaßen ausgedrückt werden: 


"ich bin genötigt, sonst..."
"eine mir bekannte Instanz (MAN,
DOXA oder Person $\mathrm{D}_{\text {individ }}$ ) stellt ein
Gesetz auf, demzufolge p eine
notwendige Bedingung für q ist (ohne
$\mathrm{p}$ kein q erreicht werden kann); ich
will q, daher will ich p"

"ich bin bloß genötigt zu ..., und ich werde ..."

"eine mir bekannte Instanz (MAN, DOXA oder Person $\mathrm{D}_{\text {individ }}$ ) stellt ein Gesetz auf, demzufolge $p$ eine hinreichende Bedingung für $q$ ist ( $p$ alleine bereits $\mathrm{zu} \mathrm{q}$ führt); ich will q, daher will ich p"

\begin{abstract}
“es gibt jemanden (darunter mich), der genötigt ist, sonst ..."

"eine mir bekannte Instanz (MAN, DOXA oder Person $\mathrm{D}_{\text {individ }}$ ) stellt ein Gesetz auf, demzufolge es mindestens eine Person $\mathrm{X}$ gibt, für die gilt, dass $\mathrm{p}$ eine notwendige Bedingung für $\mathrm{q}$ ist (ohne p kein q erreicht werden kann); ich bin eine solche Person X; ich will $\mathrm{q}$, daher will ich $\mathrm{p}^{\prime \prime}$
\end{abstract}

“es gibt jemanden (darunter mich), der bloß genötigt ist zu ..., und ich werde" "eine mir bekannte Instanz (MAN, DOXA oder Person $D_{\text {individ }}$ ) stellt ein Gesetz auf, demzufolge es mindestens eine Person $\mathrm{X}$ gibt, für die gilt, dass $\mathrm{p}$ eine hinreichende Bedingung für $\mathrm{q}$ ist (p alleine bereits zu q führt); ich bin eine solche Person $X$; ich will q, daher will ich $\mathrm{p}^{\prime \prime}$

Diese Raisonnements können, wie wir in Kratschmer (1998) und (2005c) gezeigt haben, formalisiert werden, indem man sie als Syllogismen aufstellt, wo die einzelnen Prämissen jeweils mit ihrem eigenen Modus ausgestattet sind (der Sender ist sich der Gesetzmäßigkeit bewusst, daher ist der zur praemissa maior gehörige Modus "denken"; mit der praemissa minor und der Konklusion ist dahingegen ein volitiver Modus verbunden):

Sender-denken $(\neg \mathrm{p} \rightarrow \neg \mathrm{q})^{22}$

Sender-wollen (q)

Sender-wollen (p) 
Mittlerweile sind wir jedoch der Ansicht, dass man diese Raisonnements in vorteilhafter Weise in polyphone Konfigurationen konvertieren kann, indem man die einzelnen Propositionen verschiedenen Diskursindividuen zuschreibt. Damit erlangt man größere Einsicht darüber, wie der Sender in seiner Rolle als Textkonstrukteur LOC die Verantwortung für die einzelnen Propositionen (welche zu Standpunkten werden) verschiedenen Bildern von (reellen oder imaginären) Gesprächspartnern (Diskursindividuen) zuschreibt.

Der Textkonstrukteur LOC schreibt die Gesetzmäßigkeit jener Instanz zu, welche als Quelle für diese angesehen wird, das heißt, je nach Situation, dem teilbaren kollektiven MAN, dem unteilbaren kollektiven DOXA oder einer individuellen Person, welche eine dritte Person oder der Gesprächspartner oder - in bestimmten Fällen - der Sender selbst sein kann.

Der Wunsch, das Endziel (q) zu erreichen, wird dem Sender in seiner Rolle als historisches Wesen zugeschrieben, als Wesen, welches einen Standpunkt über längere Zeit erworben und beibehalten hat (S): der Wunsch, das Endziel zu erreichen, kann als etwas relativ Konstantes angesehen werden, als etwas, das den Hintergrund für das gesamte Raisonnement bildet.

Der Wunsch, das "Zwischenziel" (die Bedingung für q, in Form von p) zu erreichen, kann dem Sender in seiner Rolle als ad hoc-Sender $\left(\mathrm{s}_{0}\right)$ zugeschrieben werden, einem Senderbild, welches nur in der aktuellen Aussage existiert, wo es hier und jetzt zu erkennen gibt, p zu wollen ("ich bin zu p genötigt"). 


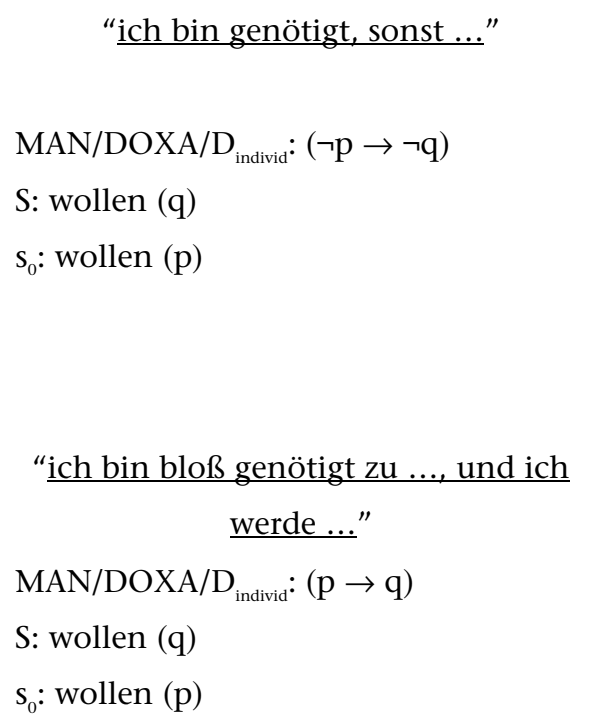

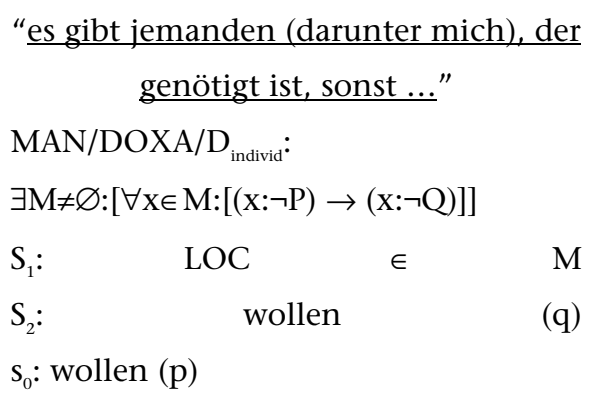

"es gibt jemanden (darunter mich), der bloß genötigt ist $\mathrm{zu} . . .$, und ich werde" $\mathrm{MAN} / \mathrm{DOXA} / \mathrm{D}_{\text {individ }}$ : $\exists \mathrm{M} \neq \varnothing:[\forall \mathrm{x} \in \mathrm{M}:[(\mathrm{x}: \mathrm{P}) \rightarrow(\mathrm{x}: \mathrm{Q})]]$ $S_{1}: L O C \in M$ $\mathrm{S}_{2}$ : wollen (q) $\mathrm{s}_{0}$ : wollen $(\mathrm{p})$

Für den Fall des paradigmatisierten genötigten Subjekts muss angemerkt werden, dass es hier noch einen Standpunkt gibt, nämlich jenen, welcher konstatiert, dass sich die Gesetzmäßigkeit auf den Sender bezieht. Wir meinen, dass es natürlich ist, diesen Standpunkt ebenfalls dem Senderbild S ("Wesen mit Geschichte") zuzuschreiben, da es hier um die Einsicht des Senders selbst geht, in die Gesetzmäßigkeit involviert zu sein, welche erneut Hintergrund für das Raisonnement ist. $S$ wird daher von den Teilbildern $S_{1}$ und $S_{2}$ repräsentiert, doch ohne dass die Zahlindexe intendiert wären, eine zeitliche oder andere Priorität auszudrücken.

Wenn man nun erneut diese Resultate in Bezug auf die semantischen Rollen der involvierten dativischen Größen beurteilt, dann kann man feststellen, dass bei den deontisch/alethischen Prädikaten der Unterschied zwischen den Kontexten mit mi/a me auf der einen und per me auf der anderen Seite nicht im Einbeziehen der gesetzgebenden Instanz $M A N / D O X A / D_{\text {individ }}$ besteht: Diese Instanz ist in beiden Fällen vorhanden. Der Unterschied besteht in der Bezugnahme des Gesetzes auf eine bestimmte Person " $\mathrm{X}$ " (Paradigmatisierung), welche

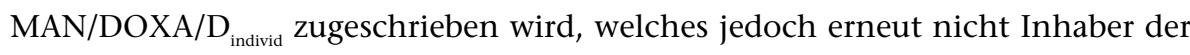
dativischen semantischen Rolle sein kann, da dieses Diskursindividuum nicht 
koreferent mit dem modalen Subjekt io "ich" (das heißt dem Sender) ist. Wie bei den epistemischen Prädikaten kann man auch hier feststellen, dass die dativische semantische Rolle Interpretationsinstruktionen für eine ganze polyphone Konfigurationen gibt und nicht mit einem einzelnen Diskursindividuum assoziiert werden kann.

\subsection{Scheinen-Prädikate}

Wie bei den epistemischen und deontisch/alethischen Prädikaten beginnen wir mit einer Paraphrase der Scheinen-Kontexte, welche das modale Subjekt als grammatikalisches Subjekt aufweist. Wir gehen zunächst von zwei sehr allgemein gehaltenen Paraphrasen aus, welche für alle Konstruktionstypen gelten und deren linke Version sich auf Scheinen-Kontexte mit dativischer Ergänzung und deren rechte Version sich auf solche ohne dativische Ergänzung bezieht:

\section{Das beurteilende Subjekt}

Mi sembra $\cong($ io $)$ mi sono fatto un Sembra $\cong($ io $)$ mi sono fatto un giudizio giudizio sulla base di informazioni $X, m a$ può darsi che si debba rivedere ad un certo punto più avanti nel tempo

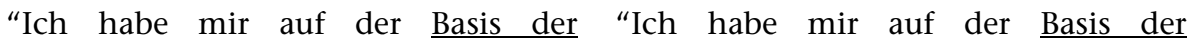
Informationen $\mathrm{X}$ ein Urteil gebildet, Informationen $\mathrm{Y}$ ein Urteil gebildet, welches womöglich zu einem späteren Zeitpunkt revidiert werden muss" sulla base di informazioni $Y$, ma può darsi che si debba rivedere ad un certo punto più avanti nel tempo welches womöglich zu einem späteren Zeitpunkt revidiert werden muss"

Im Anschluss daran bringen wir nun unsere Paraphrasen für die einzelnen Konstruktionstypen, da letztere ja unterschiedliche semantische Nuancen, namentlich bezüglich des evidentiellen Bereichs, vehikulieren: 
$\underline{\text { Kompletivsatz }}$

(mi) sembra che ("mir scheint, dass")

"ich habe mir ein Urteil gebildet indem ich auf der Basis von eigenen

$\underline{\text { Indizien }}$ eine Konklusion gezogen habe; dieses Urteil muss jedoch womöglich $\mathrm{zu}$ einem

Zeitpunkt revidiert werden"

Raising

Gianni (mi) sembra scuotere le spalle

("Gianni scheint mir mit den

Schultern zu zucken") $\underline{\text { Kompletivsatz }}$

(*mi) sembra che ("Es heißt", "Es

scheint, dass")

"ich habe mir ein Urteil gebildet, indem ich auf ein Gerücht gehört habe; dieses Urteil muss jedoch womöglich zu einem späteren Zeitpunkt revidiert werden"

"ich habe mir ein Urteil gebildet, indem ich auf der Basis von fremden Indizien eine Konklusion gezogen habe; dieses Urteil muss jedoch womöglich $\mathrm{zu}$ einem späteren Zeitpunkt revidiert werden"

$\underline{\text { Raising }}$

Gianni (*mi) sembra essere malato

("Gianni scheint (*mir) krank zu sein")

Quest'idea (*mi) sembra essere nuova

("Diese Idee scheint (*mir) neu zu sein")

"ich habe mir ein Urteil gebildet, "ich habe mir ein Urteil gebildet, indem ich auf der Basis von eigenen indem ich auf der Basis von eigenen $\underline{\text { Indizien }}$ eine Konklusion bezüglich eines durch physische Perzeption zugänglichen Sachverhaltes gezogen habe; dieses Urteil muss jedoch womöglich $\mathrm{zu}$ einem späteren Zeitpunkt revidiert werden" oder fremden Indizien eine

$\underline{\text { Konklusion bezüglich eines nicht }}$ durch physische Perzeption zugänglichen Sachverhaltes gezogen habe; dieses Urteil muss jedoch womöglich $\mathrm{zu}$ einem späteren Zeitpunkt revidiert werden" 


\section{Small clause}

Gianni (mi) sembra malato.

("Gianni scheint mir krank (zu sein)")

Quest'idea (mi) sembra nuova.

("Gianni scheint mir neu (zu sein)")

"ich habe mir ein unmittelbares

perzeptives oder intellektuelles Urteil

gebildet; dieses Urteil muss jedoch

womöglich $\mathrm{zu}$ einem späteren

Zeitpunkt revidiert werden"

In Kratschmer (2005a) bzw. (2005b) haben wir unter dem Hinweis auf den Gebrauch ein und desselben Lexems in verschiedenen, jedoch miteinander verwandten Lesarten für eine parallele polyphone Analyse all dieser Kontexte argumentiert, wo der Sender in seiner Rolle als Diskursindividuum, welches nur in der aktuellen Aussage existiert $\left(\mathrm{s}_{0}\right)$, hic et nunc ein epistemisches Urteil bezüglich des in der untergeordneten Prädikation ausgedrückten Sachverhaltes $\mathrm{p}$ abgibt. Dieses epistemische Urteil lautet jeweils "p ist meine vorläufige Konklusion". Die Verantwortung für die Assertion des Sachverhaltes p, zu welchem $\mathrm{s}_{0}$ hic et nunc Stellung nimmt und welche daher den Hintergrund für den Kommentar durch $s_{0}$ bilden muss, wird - mit Ausnahme der Gerücht-Lesart (s. im Anschluss) - dem Sender in seiner Rolle als historisches Wesen, welches einen Standpunkt über längere Zeit erworben und beibehalten hat (S), zugeschrieben. Es handelt sich daher um Kontexte interner Polyphonie:

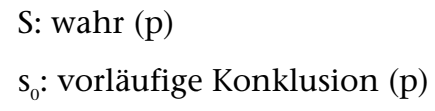

In der Gerücht-Lesart, welche durch die Kompletivsatzkonstruktion vehikuliert werden kann, wird der Standpunkt $\mathrm{p}$ dem kollektiven DrittepersonDiskursindividuum MAN zugeschrieben, wodurch eine Konfiguration externer Polyphonie aufgebaut wird:

MAN: wahr (p)

$\mathrm{s}_{0}$ : vorläufige Konklusion $(\mathrm{p})$ 
Will man nun erneut versuchen, durch den Vergleich der einzelnen polyphonen Konfigurationen zu überprüfen, welches Diskursindividuum eventuell mit der dativischen semantischen Rolle der mi-Ergänzungen in den Scheinen-Kontexten zu assoziieren wäre, so wird erneut deutlich, dass dies nicht möglich ist: mit Ausnahme der Gerücht-Lesart weisen alle Konstruktionen die gleiche polyphone Konfiguration auf und jene ist daher erneut in ihrer Gesamtheit mit der dativischen semantischen Rolle des beurteilenden Subjekts verbunden.

Darüber hinaus beleuchten die Scheinen-Kontexte noch eine weitere, interessante Informationsvernetzung. Wie in Abschnitt 4.3. gezeigt, ist die dativogene Natur der Scheinen-Kontexte in starkem Maße an evidentielle Gegebenheiten gebunden: So schließt etwa in Bezug auf Kompletivsatz- und raisingKonstruktionen das Heranziehen fremder Indizien für eigene Inferenzen die dativischen Ergänzungen aus. Die polyphone Formalisierung kann der Evidentialität keine Rechnung tragen (erneut mit Ausnahme der Gerücht-Lesart, wo die externe Quelle MAN für $\mathrm{p}$ automatisch einen evidentiellen Indikator darstellt), beide Beschreibungbereiche können jedoch als interagierende Module betrachtet werden. Wir argumentieren hier im Sinne der modulären Linguistik nach Nølke, welche für die Erstellung von Mini-Theorien für begrenzte Forschungsgebiete plädiert, die in einem zweiten synthetischen Schritt zu einem globalen organischen System vereinigt werden, dessen Module sie sodann ausmachen. In diesem Sinne sind polyphone und evidentielle Aspekte jeweils in einem anderen Modul $\mathrm{zu}$ bearbeiten, wobei die Module jedoch über "Metaregeln" interagieren (vgl. etwa Nølke 1994). In den Scheinen-Kontexten speist das evidentielle Modul, welches die Informationsbeschaffungsvorgänge verwaltet, das polyphone Modul mit dem Ergebnis der Informationsrequirierung, welches dieses sodann als Standpunkt von S bzw. MAN konfiguriert und von $s_{0}$ kommentieren lässt. Dies bedeutet, dass der dativogene Charakter der Scheinen-Kontexte nicht nur, wie dies bei den rein epistemischen und den deontisch-alethischen Kontexten der Fall war, mit der jeweiligen polyphonen Gesamtkonfiguration in Zusammenhang steht, sondern dazu auch Zugang zum evidentiellen Modul hat.

\section{Die personelle und temporale Perspektive}

Unsere Analyse der Semantik der endosubjektiven Prädikate war bis hierher auf Kontexte beschränkt, wo das modale Subjekt in der ersten Person stand, obwohl 
einige unserer weiter oben genannten empirischen Beispiele modale Subjekte in der zweiten, z.B. (44), oder dritten Person, z.B. (45), aufgewiesen hatten. Die Ursache dafür ist ausschließlich im Wunsch nach einer einfachen, übersichtlichen Präsentation zu suchen, stellt jedoch in keinster Weise ein theoretisches Problem dar. Wie in Kratschmer/Nølke (2005) gezeigt wurde, können polyphone Konfigurationen, welche Sender-Diskursindividuen vom Typus $\mathrm{S}$ und $\mathrm{s}_{0}$ enthalten, in die zweite (= Empfänger) und dritte Person transponiert werden, da ScaPoLines neueste Versionen (vgl. etwa Nølke 2005b) die Existenz von Zweite- und Dritteperson-Diskursindividuen vorhersehen, welche sich ebenfalls in Bilder "Wesen mit Geschichte" $\left(\mathrm{E}^{23}\right.$ und $\left.\mathrm{D}^{24}\right)$ und ad hocBilder, welche nur in der aktuellen Aussage existieren $\left(e_{0}\right.$ und $\left.d_{0}\right)$, einteilen lassen.

Dies kann folgendermaßen illustriert werden:

$$
\begin{aligned}
& \text { mi è chiaro "mir ist klar" } \\
& \text { "ich weiß, dass p" }
\end{aligned}
$$

MAN: wahr (p) [objektivierende

Instanz]

S: wahr (p)

$\mathrm{s}_{0}$ : wahr (p)

mi è necessario "mir ist notwendig" > >

"ich bin genötigt, sonst ..."

MAN/DOXA/D individ $:(\neg p \rightarrow \neg q)$

S: wollen (q)

$\mathrm{s}_{0}$ : wollen (p)

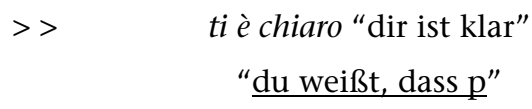

MAN: wahr (p) [objektivierende

Instanz]

E: wahr (p)

$\mathrm{e}_{0}$ : wahr $(\mathrm{p})$

gli è necessario "ihm ist

notwendig"

"er ist genötigt, sonst ..."

MAN/DOXA/ individ $_{\text {in }}(\neg p \rightarrow \neg q)$

D: wollen (q)

$\mathrm{d}_{0}$ : wollen $(\mathrm{p})$

Darüber hinaus können polyphone Konfigurationen zeitlich transponiert werden (siehe ebenfalls Kratschmer/Nølke 2005), sodass Vergangenheitskontexte auf gleiche Art und Weise wie die bisher analysierten Gegenwartskontexte formalisiert werden können: 
gli è necessario "ihm ist notwendig" >>

"er ist genötigt, sonst ..."

MAN/DOXA/D $D_{\text {individ }}:(\neg p \rightarrow \neg q)$

D: wollen (q)

$\mathrm{d}_{0}$ : wollen $(\mathrm{p}), \mathrm{t}_{0}$ gli era necessario "ihm war notwendig"

"er war genötigt, sonst ..." MAN/DOXA/D individ $:(\neg p \rightarrow \neg q)$

D: wollen $(\mathrm{q})$

$\mathrm{d}_{\mathrm{t}}$ : wollen $(\mathrm{p}), \mathrm{t}_{\mathrm{t}}$

Die Abkürzung " $\mathrm{d}_{0}$ " steht im Übrigen für das ad hoc-DrittepersonDiskursindividuum zum gegenwärtigen Aussagezeitpunkt (Zeitpunkt " $\mathrm{t}_{0}$ "; wir nennen " $\mathrm{d}_{0}$ " daher hic et nunc-Diskursindividuum), während " $\mathrm{d}_{\mathrm{t}}$ " für das ad hocDritteperson-Diskursindividuum zum vergangenen Aussagezeitpunkt (Zeitpunkt " $\mathrm{t}_{\mathrm{t}}$ "; wir nennen " $\mathrm{d}_{\mathrm{t}}$ " daher ibi et tunc-Diskursindividuum) steht.

\section{KATEGORIEN EPISTEMISCHER BeURTEILUNG UND DIE PLATZIERUNG VON È EVIDENTE, È CHIARO}

Wir verdanken Hans Kronning die Beobachtung, dass es verschiedene Kategorien epistemischer Beurteilung gibt. Kronning (2005: 82) definiert auf der einen Seite die epistemische Attitüde als "mentalen Zustand eines kognitiven Subjekts": Peter ist sicher/unsicher. Dahingehend bezeichnet epistemische Modalität bei Kronning (2005: 76) die "Stärke", welche ein modales Subjekt einem Wissensinhalt zuteilt ("wahr", "wahrscheinlich", "notwendigerweise wahr"). Die epistemische Modalität kann kein kognitives Subjekt charakterisieren: *Peter ist (un)wahrscheinlich.

Versucht man nun die epistemischen Ausdrücke, welche wir bisher untersucht haben, mit und ohne dativische Ergänzungen, diesen beiden Beurteilungskategorien zuzuordnen, so kann man zunächst anführen, dass è indubbio "es ist zweifelsfrei" und è certo "es ist gewiss" als Ausdrücke der epistemischen Modalität klassifiziert werden können, das heißt, dass sie etwas über die "Stärke" (den Wahrscheinlichkeitsgrad) aussagen, welche das modale Subjekt einem Sachverhalt zuteilt.

Sodann können unsere Paraphrasen für è evidente "es ist deutlich" und è chiaro "es ist klar" in Kombination mit dativischen Ergänzungen, welche (io) so "ich weiß" vs. (io) sono convinto "ich bin überzeugt" lauten, als Ausdrücke der 


\section{DER VERFLIXTE ITALIENISCHE DATIV - EIN (ERSTER) SEMANTISCH-GRAMMATIKALISCHER \\ ZÄHMUNGSVERSUCH}

epistemischen Attitüde klassifiziert werden, das heißt als Ausdrücke, welche den mentalen Zustand eines kognitiven Subjekts betreffen.

Die beiden Ausdrücke è evidente und è chiaro ohne dativische Ergänzungen können dahingegen keiner der bisher genannten Kategorien zugeordnet werden: *Peter ist evident ergibt keinen Sinn (es handelt sich daher nicht um epistemische Attitüde). Auf der anderen Seite drücken die beiden Formen auch keine Wahrscheinlichkeitsbeurteilung aus.

Die beiden Formen gehören einer dritten Kategorie epistemischer Beurteilung an, einer Kategorie, welche wir "epistemische Prädikation" nennen wollen, da diese in einer sekundären Prädikation (oder Prädikation zweiten Grades) über eine Basisprädikation (den untergeordneten propositionellen Inhalt) besteht. Die epistemische Prädikation definiert die epistemische Zugänglichkeit der untergeordneten Prädikation (Zugänglichkeit für das kognitive Subjekt) und präsentiert diese epistemische Zugänglichkeit als eine inhärente ("objektive") Eigenschaft des Sachverhalts selbst: Wenn man sagt è chiaro che non piove "es ist klar, dass es nicht regnet", dann präsentiert man den Sachverhalt "nicht-Regen" als einen Sachverhalt mit der inhärenten Eigenschaft "(epistemische) Klarheit", das heißt einen Grad an epistemischer Zugänglichkeit. ${ }^{25}$

Das Interessante ist nun, dass das Hinzufügen von dativischen Größen zu ̀̀ evidente und è chiaro einen Kategoriewechsel auslöst: die Lesart wechselt von epistemischer Prädikation zu epistemischer Attitüde: è chiaro "es ist klar" > > mi è chiaro "ich weiß" und per me è chiaro "ich bin überzeugt". Eine dativische Ergänzung, welche bei einigen dativogenen Prädikaten ausschließlich die Subjektivität einer bereits subjektiven Beurteilung zu verstärkten scheint (sembra "es scheint" > > mi sembra "mir scheint", wie Henning Nølke und Verf. im Rahmen einer Diskussion festgestellt haben), vermag eine epistemische Prädikation in eine epistemische Attitüde umzuwandeln, das heißt, als "Subjektivator" zu fungieren, als Element, welches eine "objektive" Beurteilung subjektiviert. 


\section{SCHLUßFOLGERUNGEN}

\subsection{Praktische (sprachdidaktische) Konsequenzen}

Bezüglich einiger bestimmter italienischer epistemischer (è evidente "es ist deutlich", ’̀ chiaro "es ist klar") und deontisch/alethischer Prädikate (occorre, bisogna, necessita, è necessario "es ist notwendig"; ’̀ obbligatorio "es ist verpflichtend"; basta "es ist hinreichend") konnte ein semantischer Unterschied zwischen Prädikaten mit Ergänzungen in Form von dativischen klititischen Pronomen oder durch die Präposition $a$ eingeleiteten Präpositionalphrasen auf der einen Seite und Prädikaten mit Ergänzungen in Form von durch die Päposition per eingeleiteten Präpositionalphrasen auf der anderen Seite festgestellt werden.

Im epistemischen Bereich kann man etwa unterscheiden zwischen mi è chiaro und a me è chiaro (wörtlich "mir ist klar"), welche eine Lesart (io) so "ich weiß" haben, und per me è chiaro "für mich ist klar", welches eine Lesart (io) sono convinto "ich bin überzeugt" hat. Das heißt, dass sich die Semantik des Prädikats selbst mit der Kategorie der Ergänzung ändert.

Im deontisch/alethischen Bereich liegen die Unterschiede hingegen in der Präsentation des dativischen modalen Subjekts als entweder absolut oder mittels Emphase paradigmatisiert. So kann man etwa unterscheiden zwischen mi è necessario und a me è necessario (wörtlich "mir ist notwendig"), welche eine Lesart (io) [ausgelassen oder schwach betont] sono costretto "ich bin genötigt" haben, und per me è necessario "für mich ist (es) notwendig", welches eine Lesart $\underline{i o}$ [mit emphatischer Intonation] sono costretto "es gibt jemanden (darunter mich), der genötigt ist" hat.

Im pronominalen Bereich, wo sowohl klitische, unbetonte Pronomen (mi/ti/gli "mir"/"dir"/"ihm") als auch betonte, von der Präposition a abhängige, Pronomen (a me/a te/a lui "mir"/“dir"/“ihm") zur Verfügung stehen, stehen diese in einer informationsstrukturellen Opposition: $a+$ betontes Pronomen markiert das thematisierte modale Subjekt, während das klitische Pronomen das nichtthematisierte modale Subjekt markiert. Im epistemischen Bereich besteht die Opposition zwischen dem thematisierten und dem nicht thematisierten wissenden Subjekt, im deontisch/alethischen Bereich zwischen dem 


\section{DER VERFLIXTE ITALIENISCHE DATIV - EIN (ERSTER) SEMANTISCH-GRAMMATIKALISCHER ZÄHMUNGSVERSUCH}

thematisierten und dem nicht-thematisierten absoluten (nicht emphatischen) genötigten Subjekt.

Alle muttersprachlichen Informanten klassifizierten è evidente "es ist deutlich" + Dativpronomen mi/gli als nicht akzeptabel, è evidente $+a+$ Indefinitpronomen tutti "alle" als normkonform, während die Meinungen bezüglich è evidente $+a+$ betontes persönliches Pronomen me geteilt waren.

Bezüglich der Kombination von bisogna ("es ist notwendig") mit dativischen Ergänzungen sei erwähnt, dass, je nach Alter der Informanten, diese Verbindungen entweder als gängig oder als archaisch (nicht länger normkonform) beurteilt wurden.

Das Prädikat ̀̀ obbligatorio "es ist verpflichtend" kann nicht mit einer $a$ Präpositionalphrase mit nominalem Regimen kombiniert werden, sondern nur mit einer per-Präpositionalphrase, wodurch die per-Formen ihre emphatische Lesart verlieren. Dahingegen existiert im pronominalem Bereich die bekannte Opposition zwischen dem absoluten und dem paradigmatisierten, emphatischen genötigten Subjekt.

Für alle Scheinen-Prädikate (sembrare und parere) in allen Konstruktionen gilt, dass sie, sofern sie dativische Ergänzungen zulassen, diese nur in Form von echten dativischen klitischen Pronomen (mi, ti, gli etc. "mir", "dir", "ihm") oder in Form von mit $a$ eingeleiteten Präpositionalphrasen ( $a$ me "mir", all'insegnante "dem Lehrer") akzeptieren. Mit per eingeleitete Präpositionalphrasen sind ungrammatisch. Kompatibel mit dativischen Ergänzungen sind alle small clauseKonstruktionen, Kompletivkonstruktionen, die eine vorangegangene Inferenzleistung auf der Basis von eigenen Indizien ausdrücken, sowie raisingKonstruktionen, welche ebenfalls eine vorangegangene Inferenzleistung auf der Basis von eigenen Indizien ausdrücken, allerdings nur in jenem Fall, wo der zu beurteilende Sachverhalt dem Sprecher direkt perzeptiv zugänglich ist. Nicht kompatibel mit dativischen Ergänzungen sind Kompletivkonstruktionen mit Gerücht-Lesart und solche, die eine vorangegangene Inferenzleistung auf der Basis von fremden Indizien vehikulieren, und alle übrigen raisingKonstruktionen (dies wären sowohl jene, welche eine vorangegangene Inferenzleistung auf der Basis von fremden Indizien ausdrücken, und schließlich 
jene, die eine vorangegangene Inferenzleistung auf der Basis von eigenen Indizien kodifizieren, wo der zu beurteilende Sachverhalt dem Sprecher jedoch nicht direkt perzeptiv zugänglich ist).

\subsection{Theoretische Konsequenzen}

In Bezug auf eine Annäherung an eine konzise Definition des Begriffs "semantischer Dativ" und die dazu notwendige systematische Beschreibung und Klassifikation der "dativischen" semantischen Rollen, welche bestimmte Satzglieder innehaben können, können wir als einen ersten Schritt auf diesem Wege notieren, dass für die bisher untersuchten italienischen "endosubjektiven" Prädikate (Prädikate, welche eine neurokognitive Gegebenheit in einem bewussten Subjekt ausdrücken) gilt, dass diese keineswegs überraschend - eine polyphone Analyse zulassen. Im Rahmen einer solchen polyphonen Analyse kann der Inhalt dieser Prädikate als eine polyphone Konfiguration formalisiert werden, innerhalb derer eine Reihe an Diskursindividuen als Quellen für ihre je eigenen Standpunkte präsentiert werden. Diese Diskursindividuen sind typischerweise Bilder des modalen Subjektes in dessen zwei Ausformungen ( $\mathrm{S}$ und $\mathrm{s}_{0 / t} ; \mathrm{E}$ und $\mathrm{e}_{0 / t} ; \mathrm{D}$ und $\mathrm{d}_{0 / /}$ ), meist in Kombination mit einem Dritteperson-Diskursindividuum des Typs MAN/DOXA. Im Gegensatz zu unserer ursprünglichen Hypothese kann der Unterschied in Bezug auf die semantische Rolle der dativischen Ergänzungen zu den endosubjektiven Prädikaten jedoch nicht einem einzelnen Diskursindividuum innerhalb der polyphonen Konfiguration zugeschrieben werden, sondern muss vielmehr in jenem Licht betrachtet werden, dass die verschiedenen semantischen Rollen unterschiedliche Interpretationsinstruktionen bezüglich verschiedener - polyphoner Gesamtkonfigurationen zur Verfügung stellen. Diese Gesamtkonfigurationen sind verschieden, je nachdem, ob die dativischen Ergänzungen die Form von Dativpronomen und $a$-Präpositionalphrasen auf der einen Seite oder, auf der anderen Seite, von per-Präpositionalphrasen haben. Die epistemischen Prädikate beziehen im ersten Fall eine objektivierende Instanz MAN/DOXA in ihre polyphone Konfiguration mit ein, welche im zweiten Falle fehlt. Die deontisch/alethischen Prädikate beinhalten in beiden Fällen eine gesetzgebende Instanz, welche wiederum MAN/DOXA sein kann, aber auch z.B. eine individuelle (erste, zweite, dritte) Person. Das Gesetz wird jedoch im ersten Fall als allgemeingültig präsentiert, im zweiten Fall dahingegen als sich auf das modale Subjekt beziehend. In Bezug auf den dativogenen Charakter der Scheinen- 


\section{DER VERFLIXTE ITALIENISCHE DATIV - EIN (ERSTER) SEMANTISCH-GRAMMATIKALISCHER ZÄHMUNGSVERSUCH}

Kontexte konnten wir zeigen, dass dieser nicht nur, wie dies bei den rein epistemischen und den deontisch/alethischen Kontexten der Fall war, mit der jeweiligen polyphonen Gesamtkonfiguration in Zusammenhang steht, sondern dazu auch mit evidentiellen Gegebenheiten verbunden ist: dativische Ergänzungen sind nur in jenen Fällen zugelassen, wo entweder ein direktes, perzeptives oder intellektuelles, Urteil abgegeben wird (small clauseKonstruktionen) oder eine Beurteilung, welche Resultat einer Inferenz ist, welche sich auf eigene (und nicht auf fremde) Indizien stützt (Kompletiv- und raisingKonstruktionen, wobei bei letzteren zudem noch das Kriterium erfüllt sein muss, dass die untergeordnete Prädikation einen Sachverhalt ausdrückt, der dem Sender direkt perzeptiv zugänglich ist).

Die epistemischen Prädikate è evidente und ̀̀ chiaro gehören einer bestimmten Unterkategorie an epistemischen Beurteilungen an, namentlich der Kategorie "epistemische Prädikation" (eine sekundäre Prädikation, welche die epistemische Zugänglichkeit einer Prädikation als eine inhärente, "objektive" Eigenschaft des ausgedrückten Sachverhaltes selbst präsentiert). Das Hinzufügen von dativischen Größen zu è evidente und è chiaro löst jedoch einen Kategoriewechsel aus: die Konstruktionen drücken dann keine epistemische Prädikation mehr aus, sondern vielmehr eine epistemische Attitüde (das heißt einen mentalen Zustand eines kognitiven Subjekts): è chiaro "es ist klar" > > mi è chiaro "ich weiß" und per me è chiaro "ich bin überzeugt".

Darüber hinaus zeigte die Korpusarbeit, dass die Ergänzungen in Form von dativischen Pronomen und von $a$-Präpositionalphrasen typischerweise nur eine semantische Rolle per Prädikat innehaben können, während Ergänzungen in Form von per-Präpositionalphrasen sich in mehreren Lesarten (Rollen) mit ein und demselben Prädikat kombinieren lassen: z.B. auch als Zielangabe bei deontisch/alethischen Prädikaten (basta/è necessario per il successo "es ist hinreichend/notwendig für den Erfolg)" oder als vorangestellter doppelter Modus mit der Lesart (io) sono convinto "ich bin überzeugt" (il presidente è stato chiaro: per lui è necessario prendere delle misure "Der Präsident war unmissverständlich: für ihn/ihmgemäß ist es notwendig [= er ist überzeugt, dass es notwendig ist], Maßnahmen zu treffen"). Dies könnte eventuell darauf hindeuten, wie mein Kollege Svend Bach es ausdrückt, dass die Präposition $a$ ein Valenzglied einleitet, die Präposition per hingegen einen Zirkumstanten. In diese 
Richtung würden auch noch noch weitere Daten aus unserem Korpus weisen: per-Satzglieder kommen in den untersuchten Kontexten in großer Zahl auch als adverbielle Bestimmungen vor, welche nicht in direkter Verbindung mit dem endosubjektiven Bereich stehen (z.B. als Zeit-, Orts-, Art-und-WeiseErgänzungen).

\section{PeRSPEKTIVEN}

Um zu einer semantischen Definition von "dativisch" zu gelangen, ist es nach obigen Ausführungen notwendig, vergleichbare Analysen mit den übrigen italienischen dativogenen Prädikaten durchzuführen, ein Vorhaben von nicht geringem Umfang. Die festgemachten semantischen Rollen müssen dann einander gegenübergestellt werden, um die Destillation ihrer gemeinsamen Essenz zu ermöglichen.

\section{LITERATUR}

Ducrot, Oswald (1984), Le dire et le dit, Paris: Minuit.

Graffi, Giorgio (1995), Sintassi, Bologna: Il Mulino.

Grasso, Francesco (2003), 2038: la rivolta, [http://www.liberliber.it/biblioteca/ g/grasso/]

Kratschmer, Alexandra (1998), "Causalité et explication : vers une nouvelle approche", Revue Romane, 33/2 (1998), pp. 171-208.

Kratschmer, Alexandra (2005a), "Italiensk sembra/pare + kompletivsætning: modus og polyfoni", Arbejdspapirer 2, Sprogligt Polyfoninetværk, RUC, pp. 127-145.

Kratschmer, Alexandra (2005b), "Raising- og Small Clause-konstruktioner med italiensk sembrare/parere: polyfoni og evidentialitet", Arbejdspapirer 3, Sprogligt Polyfoninetværk, RUC, pp. 101-137.

Kratschmer, Alexandra (2005c), Erklärungsstrategien, semantische Felder und Makrostrukturen: eine Fallstudie zur semantischen Architektur von explikativen Texten, Habilitationsschrift, Århus, Aarhus Universitets Forlag.

Kratschmer, Alexandra (2006), "Polyphonietheoretische Formalisierung von historischen und Dritteperson-Diskursindividuen in narrativen Texten", Arbejdspapirer 5, Sprogligt Polyfoninetværk, RUC, pp. 33-63.

Kratschmer, Alexandra, Henning Nølke (2005), "Polyfone konfigurationer i narrative tekster: historiske og tredjepersonsdiskursindivider", Arbejdspapirer 4, Sprogligt Polyfoninetværk, RUC, pp. 49-94.

Kronning, Hans (2005), "Polyfoni, modalitet och evidentialitet. Om epistemiska uttryck i franskan, särskilt epistemisk konditionalis", Arbejdspapirer 3, Sprogligt Polyfoninetværk, RUC, pp. 71-99. 


\section{DER VERFLIXTE ITALIENISCHE DATIV - EIN (ERSTER) SEMANTISCH-GRAMMATIKALISCHER ZÄHMUNGSVERSUCH}

Matushansky, Ora (2002), "Tipping the Scales: The Syntax of Scalarity in the Complement of Seem", Syntax 5/3 (2002), pp. 219-276.

Nølke Henning (2001), "La focalisation énonciative : éléments d'une théorie modulaire", dans : Nølke, Henning (éd.) (2001), Le regard du locuteur 2. Pour une linguistique des traces énonciatives, Paris: Kimé, pp. 85-113.

Nølke, Henning (2005a), "The semantics of polyphony (and the pragmatics of realization)", in Acta Linguistica Hafniensia, 2006/38, pp. 137-160.

Nølke, Henning (2005b), "På vej mod en sproglig polyfoniteori - problemer, fordele, perspektiver", Arbejdspapirer 3, Sprogligt Polyfoninetværk, RUC, pp. 139-166.

Nølke, Henning, Kjersti Fløttum, Coco Norén (2004), ScaPoLine. La théorie scandinave de la polyphonie linguistique, Paris: Éditions Kimé.

Pereltsvaig, Asya (2000), "Are All Small Clauses Created Equal? Evidence from Russian and Italian", in: Yoo, M., J. Steele (eds.) (2002), McGill Working Papers in Linguistics, 15(1), pp. 73-104.

Sportiche, Dominique (1995), "French Predicate Clitics and Clause Structure", ms., in: Cardinaletti, Anna, Maria Teresa Guasti (eds.) (1995), Small Clauses, Syntax and Semantics, 1995/28, New York: Academic Press, pp. 287-324.

Das elektronische Quellenmaterial wurde im November 2005 mit Hilfe von Google beschafft, mit Ausnahme von Beispiel (36), welches vom Januar 2006 stammt (ebenfalls über Google).

\section{ANMERKUNGEN}

1 Für eine genauere Platzierung der betreffenden Ausdrücke innerhalb der übergeordneten Kategorie "epistemisches Urteil" siehe Abschnitt 8. unten.

2 Vgl. auch Kratschmer (2005a) und (2005b).

3 Wie Beispiel (49) bekräftigt ..

4 Näheres zu unserer Paraphrase "ich bin genötigt" für die deontisch/alethischen Prädikate siehe 6.2. unten.

5 Vergleiche etwa Nølkes Definition des Emphase-Phänomens als Hervorhebung einer syntaktisch abgegrenzten Informationseinheit ("Fokus") als Resultat einer Wahl innerhalb eines Paradigmas, welches ähnliche Elemente enthält; diese Hervorhebung geschieht mit einem bestimmten kommunikativen Ziel, welches der Empfänger rekonstruieren muss (Nølke 2001: 91f.).

6 Die Lexeme obbligatorio und necessitare gehören gemäß einiger muttersprachlichen Informanten in allen Kombinationen dem bürokratischen Register an, obgleich die spontane Reaktion in diese Richtung bei den Kontexten mit dativischen Pronomen und $a$-Präpositionalphrasen stärker war als bei Kontexten mit per-Präpositionalphrasen. Hier sind deutlich weitere Untersuchungen vonnöten.

7 Näheres zu unserer Paraphrase für basta "es ist hinreichend" siehe 6.2. unten.

8 Die Form bisogna "es ist notwendig" ohne dativische Ergänzungen ist dahingegen sehr gebräuchlich und registerneutral.

9 Dies ist ein Zitat des italienischen Dichters Leopardi (1798-1837). 


\section{ALEXANDRA KRATSCHMER}

10 In (43) sind a me/a te erneut als absolute, thematisierte (genötigte) Subjekte markiert.

11 Dieses Beispiel ist konstruiert, aber von den native speakern gutgeheißen.

12 Bezüglich der Kompatibilität dieser Konstruktionen mit etwa den adverbiellen Bestimmungen quasi "fast" und proprio "wirklich" sei auf Kratschmer (2005a bzw. 2005b) verwiesen.

13 Sembrare und parere präsentierten sich i. Ü. in unseren Untersuchungen den italienischen Testpersonen für alle Konstruktionstypen als absolute und damit austauschbare Synonyma (die Korpora zeigen eine frequenzmäßige Dominanz von sembrare, darüber hinaus waren bis dato keine Unterschiede festzumachen).

14 Die generative Transformationsgrammatik (vgl. z.B. Graffi 1995: 234) bezeichnet Konstruktionen wie etwa Gianni sembra essere malato ("Gianni scheint krank zu sein") als raising, da ihnen folgender Ursprung zugeschrieben wird: ein Verbalprädikat im Infinitiv (wie das hier unter sembra untergeordnete) kann seinem Subjekt keinen (Nominativ-)Kasus zuweisen; das Subjekt wird daher in die übergeordnete Subjektsposition angehoben, um dort Kasus zugewiesen zu bekommen, während die semantische (die "Theta"-)Rolle des Subjekts von der ursprünglichen Prädikation mit hinaufgenommen wird (dieser Theorie gemäß können Verben wie sembrare ihrer Subjektsposition keine eigene Thetarolle zuweisen).

15 Dieses Romanzitat hat im Original kein explizites modales Subjekt (le "ihr", mit Referenz auf die weibliche Protagonistin, aus deren Perspektive erzählt wird), ist unseren Informanten gemäß mit diesem jedoch kompatibel (Näheres zur personellen Perpektive, s. Abschnitt 7. unten).

16 Diese Konstruktionen entstehen gemäß der generativen Transformationsgrammatik ebenfalls mittels raising, jedoch mit dem Unterschied, dass die ursprüngliche untergeordnete Prädikation kein Verb enthält (daher "small clause" genannt), das heißt, dass das Prädikat von einer Nominal-, Adjektival- oder Präpositionalphrase repräsentiert wird, dessen Subjekt erneut in die Subjektsposition des übergeordneten Verbs angehoben wird, um dort seinen Nominativkasus zu erhalten. Im Rahmen des generativen Paradigmas wird diese Analyse bis zu einem gewissen Grad als Standard betrachtet, jedoch nicht ohne Ausnahme (s. z.B. Sportiche 1995, welcher das small clause-Konzept zurückweist). Unter den Anhängern herrscht darüber hinaus große Uneinigkeit bezüglich des inneren Aufbaus dieser Konstruktionen und der damit verbundenen Transformationen; s. z.B. Pereltsvaig 2000, Matushansky 2002).

17 In dessen eigener englischer Übersetzung: Joint Committee for Nordic Research Councils for the Humanities and the Social Sciences (NOS-HS).

18 In einem konkreten Kontext kann es sich dabei um den Gesprächspartner handeln, um einen bestimmten oder potentiellen Dritten. Die sprachliche Struktur gibt dazu keine Auskunft.

19 Vom französischen locuteur "Sprecher", einer der bereits von Ducrot gebrauchten Termini.

$20 \mathrm{Zu}$ den kollektiven dritten Personen zählt man u.a. das teilbare MAN, welches eine nicht näher abgegrenzte Gemeinschaft repräsentiert, und das unteilbare DOXA, welche für die Stimme des Gesetzes oder der Norm steht.

21 ScaPoLine unterscheidet die "polyphone Struktur" von der "polyphonen Konfiguration". Die Struktur ist im Sprachsystem (als Instruktionen) kodifiziert, während die Konfiguration Teil der Bedeutung der konkreten Äußerung ist. Das heißt, dass die Konfiguration teilweise von der sprachlichen Kodifizierung, teilweise aber von der Interpretation durch den Empfänger abhängt.

22 “( $\neg p \rightarrow \neg q)$ " drückt die Gesetzmäßigkeit aus, welche auf einer notwendigen Bedingung aufbaut: "ohne p kein q".

23 "E" für "Empfänger". 


\section{DER VERFLIXTE ITALIENISCHE DATIV - EIN (ERSTER) SEMANTISCH-GRAMMATIKALISCHER}

ZÄHMUNGSVERSUCH

24 "D" für "Dritte(r)".

25 Es sei jedoch erwähnt, dass die Negierung des Ausdrucks, z.B. è poco chiaro/non è chiaro "es ist wenig klar/unklar"/"es ist nicht klar", nicht länger mit einem herkömmlichen Kompletivsatz kompatibel ist, sondern einen indirekten Fragesatz verlangt: è poco chiaro se piove "es ist unklar, ob es regnet". Welche Konsequenzen dies für die epistemischen Gegebenheiten hat, bedarf weiterer Untersuchungen. 